\title{
Clustering, methodology, and mechanistic insights into acetate chemical ionization using high-resolution time-of-flight mass spectrometry
}

\author{
Patrick Brophy and Delphine K. Farmer \\ Department of Chemistry, Colorado State University, 1872 Campus Delivery, Fort Collins, CO 80523, USA \\ Correspondence to: Patrick Brophy (pbrophy@colostate.edu) and Delphine K. Farmer (delphine.farmer@colostate.edu) \\ Received: 11 May 2016 - Published in Atmos. Meas. Tech. Discuss.: 17 May 2016 \\ Revised: 24 July 2016 - Accepted: 27 July 2016 - Published: 23 August 2016
}

\begin{abstract}
We present a comprehensive characterization of cluster control and transmission through the Tofwerk atmospheric pressure interface installed on various chemical ionization time-of-flight mass spectrometers using authentic standards. This characterization of the atmospheric pressure interface allows for a detailed investigation of the acetate chemical ionization mechanisms and the impact of controlling these mechanisms on sensitivity, selectivity, and mass spectral ambiguity with the aim of non-targeted analysis. Chemical ionization with acetate reagent ions is controlled by a distribution of reagent ion-neutral clusters that vary with relative humidity and the concentration of the acetic anhydride precursor. Deprotonated carboxylic acids are primarily detected only if sufficient declustering is employed inside the atmospheric pressure interface. The configuration of a high-resolution time-of-flight chemical ionization mass spectrometer (HR-TOF-CIMS) using an acetate chemical ionization source for non-targeted analysis is discussed. Recent approaches and studies characterizing acetate chemical ionization as it applies to the HR-TOF-CIMS are evaluated in light of the work presented herein.
\end{abstract}

\section{Introduction}

Recent commercialization and packaging of time-of-flight chemical ionization mass spectrometers (TOF-CIMS) into field-deployable packages by Aerodyne Research Inc. and Tofwerk AG has led to the widespread use of these instruments (Aljawhary et al., 2013; Bertram et al., 2011; Brophy and Farmer, 2015; Chhabra et al., 2015; Ehn et al., 2010,
2011, 2014; Faust et al., 2016; Friedman et al., 2016; Jokinen et al., 2012; Junninen et al., 2010; Krechmer et al., 2015; Lee et al., 2014; Lopez-Hilfiker et al., 2016, 2015, 2014; Mohr et al., 2013; Sipilä et al., 2015; Yatavelli et al., 2012, 2014; Zhao et al., 2014). Any chemical ionization (CI) source, or more generally any near-atmospheric pressure ion source, can be installed on the front end of the mass spectrometer providing a flexible TOF instrument platform. The design and operation of the ion source affects the sensitivity of the instrument, but the fundamental ion chemistry is the key consideration to designing a CI source that is both sensitive and selective. Thus, the selection of an appropriate reagent ion for detecting the compound, or class of compounds, of interest is important (Huey, 2007). The ions observed in the TOF mass spectrum do not necessarily represent the distribution of ions generated in the ion source due to collisional dissociation (Bertram et al., 2011) and mass-dependent transmission effects (Heinritzi et al., 2016). Collisional dissociation simplifies the observed mass spectrum and has a long history of use dating back to the original developments of tropospheric CIMS measurements (Eisele, 1986). Controlling the extent of collisional dissociation can be used to investigate the ion-neutral chemistry occurring in the ion source. The TOF-CIMS uses a tunable multistate atmospheric pressure interface (API) that can eliminate or transmit clusters, but the operational details of this interface have not been investigated with systematic rigor.

TOF-CIMS represents a distinct departure from traditional quadrupole CIMS methodologies in which specific species are targeted for quantification. TOF-CIMS collects a continuous mass spectrum at high $(<1 \mathrm{~Hz})$ acquisition rates, whereas quadrupole detectors collect a limited number of 
ions due to limitations on sensitivity and time resolution due to duty cycle effects. Additionally, the high-resolution TOFCIMS (HR-TOF-CIMS) enables the assignment of a molecular formula to every observed mass peak. These two features of the HR-TOF-CIMS provide an opportunity to examine CI ion chemistry. Moreover, users can identify and observe the temporal behavior of compounds that have not previously been known to exist or calibrated in a non-targeted approach (Ehn et al., 2014). These features are, essentially, added benefits of TOF-CIMS over quadrupole CIMS instruments; however, we note that there is no substitute for authentic standard calibrations despite the alluring benefits of using TOF mass spectrometers. Quadrupole systems contrast this non-targeted approach, but calibrations remain as important for TOFs as quadrupole systems. Ideally, quadrupole CI is deployed with the intent of measuring specific species with readily available authentic calibration standards and wellcharacterized interferences. Calibrations are typically conducted for a limited number of compounds, but interferences are difficult to address until they are identified through instrument intercomparisons and careful study. Mass scans are often conducted using quadrupole-based CIMS to examine the temporal behavior of uncalibrated species, but these results are more difficult to understand compared to the mass spectrum acquired by TOF-CIMS because of the lack of elemental information present in high-resolution data. The recent identification of the decomposition of isoprene hydroxyhydroperoxides (ISOPOOH) to methyl vinyl ketone (MVK) and methacrolein (MACR) in both gas chromatograph instruments and proton-transfer reaction mass spectrometers (PTR-MS) highlights this challenge as both techniques have a long history of MVK and MACR measurements (RiveraRios et al., 2014).

Both TOF and quadrupole detectors remain subject to misinterpretation of the mass spectrum in the absence of complex interferences. Quadrupole systems with unit mass resolution can suffer from attributing the signal from a single mass to charge ratio $(\mathrm{m} / \mathrm{z})$ to a single species and potentially miss isobaric interferences at the same nominal, or unit, mass. Recent intercomparisons between co-located quadrupole PTR-MS and time-of-flight PTR-MS instruments highlight the power of high-resolution analysis in the identification of multiple overlapping peaks (Warneke et al., 2015). HR-TOF systems can separate closely spaced peaks, but knowing the actual identity of ion signals from their exact mass and extracting the high-resolution information remains challenging (Cubison and Jimenez, 2015; Stark et al., 2015). Improving the knowledge of the ion chemistry, ionization mechanisms, and instrument performance is paramount to correctly interpreting the mass spectrum because $\mathrm{CI}$ relies on selectively ionizing specific compounds or classes of compounds. Quantitative and qualitative non-targeted analysis in the complex chemical space of the atmosphere using HRTOF-CIMS necessitates characterization of the chemistry occurring within the ion source and the instrument's subsequent control over the transmission, clustering, and fragmentation of those ions.

Acetate CIMS, originally termed negative-ion protontransfer chemical ionization, is conventionally thought to selectively ionize carboxylic acids and some inorganic acids by proton abstraction (Reaction R1) (Veres et al., 2008). Other compounds, such as nitrated phenols, are detectable with acetate CIMS due to their gas-phase acidity relative to the acetate ion (Mohr et al., 2013). However, acetate can also form adducts with levoglucosan, which are detected as [levoglucosan + acetate $^{-}$clusters and not deprotonated due to their low gas-phase acidity relative to the acetate ion (Reactions R2-R3) (Zhao et al., 2014). Similarly, isoprene epoxydiols (IEPOX) and ISOPOOH have also been reported to cluster with acetate (Budisulistiorini et al., 2015). Perhaps due to this wide array of potential analytes, acetate CIMS is extensively applied to TOF-CIMS platforms under a variety of experimental configurations (Aljawhary et al., 2013; Brophy and Farmer, 2015; Budisulistiorini et al., 2015; Chhabra et al., 2015; Lopez-Hilfiker et al., 2014, 2015; Mohr et al., 2013; Wentzell et al., 2013; Yatavelli et al., 2012, 2014; Zhao et al., 2014). Using an acetate CI source coupled to the Tofwerk API, Bertram et al. (2001) demonstrated that a distribution of acetate clusters exists but can be collisionally dissociated during their transfer through the API by applying stronger electric fields across the ion optics (2011). Potential ion-molecule reactions occurring between the reagent ion and analyte $(\mathrm{R}-\mathrm{H})$ are thus as follows:

proton abstraction

$\mathrm{CH}_{3} \mathrm{C}(\mathrm{O}) \mathrm{O}^{-}+\mathrm{R}-\mathrm{H} \rightarrow \mathrm{CH}_{3} \mathrm{C}(\mathrm{O}) \mathrm{OH}+\mathrm{R}^{-}$

cluster reaction

$\mathrm{CH}_{3} \mathrm{C}(\mathrm{O}) \mathrm{O}^{-}+\mathrm{R}-\mathrm{H} \rightarrow\left[\mathrm{CH}_{3} \mathrm{C}(\mathrm{O}) \mathrm{O}+\mathrm{R}-\mathrm{H}\right]^{-}$.

We note, however, that two other types of reactions may be occurring:

ligand exchange

$\left[\mathrm{CH}_{3} \mathrm{C}(\mathrm{O}) \mathrm{O}+\mathrm{R}-\mathrm{H}\right]^{-}+\mathrm{R}^{\prime}-\mathrm{H} \rightarrow$

$\left[\mathrm{CH}_{3} \mathrm{C}(\mathrm{O}) \mathrm{O}+\mathrm{R}^{\prime}-\mathrm{H}\right]^{-}+\mathrm{CH}_{3} \mathrm{C}(\mathrm{O}) \mathrm{OH}$

declustering via collisional dissociation

$\left[\mathrm{CH}_{3} \mathrm{C}(\mathrm{O}) \mathrm{OH}+\mathrm{R}\right]^{-}+\mathrm{E}_{\mathrm{CDC}} \rightarrow \mathrm{CH}_{3} \mathrm{C}(\mathrm{O}) \mathrm{OH}+\mathrm{R}^{-}$.

While rare, fragmentation reactions are also known to occur within CIMS instrumentation:

$\mathrm{R}+\mathrm{E}_{\mathrm{CDC}} \rightarrow \mathrm{r}_{1}^{-}+\mathrm{r}_{2}$.

In light of recent studies detecting nitrated phenols as deprotonated products (Mohr et al., 2013) and detecting levoglucosan (Zhao et al., 2014) and IEPOX/ISOPOOH (Budisulistiorini et al., 2015) as acetate clusters, we suggest that these reaction should be more generalized to include other molecules with various functional groups and non-acidic 
protons. Reactions R1 and R2 have been reported in the literature for acetate CIMS assuming that carboxylic acids are detected (Bertram et al., 2011). Ligand exchange reactions (Reaction R3) have not directly been identified to occur with acetate CIMS, but the chemistry appears to be very similar to iodide adduct CIMS, where $\left[\mathrm{I}+\mathrm{H}_{2} \mathrm{O}\right]^{-}$reacts in a ligand exchange reaction with some analyte $(\mathrm{X})$ to produce $[\mathrm{I}+\mathrm{X}]^{-}$ (Lee et al., 2014). The declustering reaction (Reaction R4) is implicitly discussed by Bertram et al. (2011). Lastly, fragmentation in PTR-MS instruments is known to be an extensive feature. A study of atmospheric sesquiterpenes $\left(\mathrm{C}_{15} \mathrm{H}_{24}\right)$ identifies seven fragment ions where the specific sesquiterpene will contribute to each fragment to a different extent due to structural differences (Kim et al., 2009).

Acetate CIMS can be contrasted to iodide adduct CIMS, another rapidly developing chemical ionization method being applied to TOF-CIMS platforms (Aljawhary et al., 2013; Friedman et al., 2016; Lee et al., 2014; Lopez-Hilfiker et al., 2014, 2016; Zhao et al., 2014). Iodide adduct CIMS predominantly form iodide adducts with neutral species due to its high electronegativity; iodide is not expected to substantially abstract protons or transfer electrons (Iyer et al., 2016). Once ion-neutral clusters are formed, the ion optics of the mass spectrometer must efficiently transmit these clusters to the mass analyzer. The lack of proton abstraction or charge transfer allows this CIMS method to be operated in a cluster mode because the iodide ion holds the vast majority of the negative charge. Thus, the dominant clustering mechanism involves iodide. The dominant clustering mechanism with acetate CIMS involves the acetate ion, but the prevalence of proton abstraction produces stable anions that will also undergo clustering reactions.

The Tofwerk API enables users to control and vary the extent of collisional dissociation, allowing for more representative descriptions of ion source chemistry. The Tofwerk API consists of two segmented radio-frequency-only (RF) quadrupoles: the Short Segmented Quadruple (SSQ) and the Big Segmented Quadrupole (BSQ). These components are housed in two differentially pumped vacuum chambers and contain various skimmers and entrance plates (Fig. 1). Between the entrance of the API and the last skimmer after the $\mathrm{BSQ}$, there are nine individually controllable voltage components and the two RF-only segmented quadrupoles, making the task of optimally tuning the API a serious undertaking. This task is made more complex by the realization that instrument resolution, ion transmission efficiency, and extent of collisional dissociation are all interrelated.

Previous studies have examined these components with regard to controlling and/or understanding the transmission of ions and clusters to the TOF (Bertram et al., 2011; Heinritzi et al., 2016). The original description of the Tofwerk API uses no ion source and describes ambient ions and ionneutral clusters in the atmosphere (Junninen et al., 2010). The authors characterize the ion transmission efficiency of the API-TOF tuned to transmit clusters using an electrospray

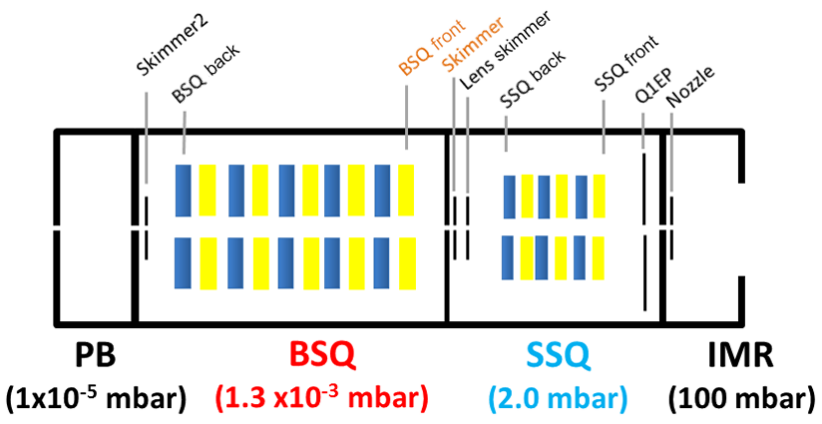

Figure 1. Schematic of the Tofwerk atmospheric pressure interface (API) showing where the IMR mounts on the API, the short segmented quadrupole (SSQ), the big segmented quadrupole (BSQ), and the primary beam $(\mathrm{PB})$ region.

source emitting tetraheptylammonium bromide. Ion transmission efficiency is defined as the fraction of ions at the inlet or in the ion source that make it to the detector. No attempt to systematically characterize or optimize the API components is presented, although the authors note that ion transmission efficiency is strongly dependent on the voltage settings in the API. A comparison of methods for experimentally determining mass-dependent ion transmission efficiency has also been reported, but no evaluation of the voltage settings, their relationships, and their effect on clustering or transmission efficiency is reported (Heinritzi et al., 2016).

The application of the Tofwerk API to a C-TOF configured as an acetate $\mathrm{CI}$ instrument provides some more insight to understanding the relationships between various API components as they relate to cluster transmission and collisional dissociation (Bertram et al., 2011). Here, the authors suggest that collisional dissociation of ion-neutral clusters occurs between the exit of the SSQ and the entrance of the BSQ vacuum stage. This claim appears to be in slight contrast to the recent results describing the use of voltage scanning to determine instrument sensitives with the iodide reagent ion (Lopez-Hilfiker et al., 2016). The authors scan the API voltages to increase the difference between the BSQ front and the last skimmer after the SSQ and lens skimmer (Fig. 1 and Table 1). Ion transmission efficiency is maintained by floating all components upstream of the last skimmer more negative as the voltage difference between these two components is increased. Systematic floating of API components to change the voltage difference between two components maintains the electric field strengths between all other components. This approach also prevents changing the axial-electric field across the RF-only segmented quadrupoles; changing the axial-electric field will result in changes in ion transmission efficacy which must be avoided so that a mass spectrum collected under one voltage setting is comparable to results collected using a different voltage configuration.

Previous work comparing the iodide adduct, acetate, and water cluster CIMS methodologies using a HR-TOF-CIMS 
Table 1. Component relationships defined by adjacent components in the API (see Fig. 1 for API schematic).

\begin{tabular}{lll}
\hline $\begin{array}{l}\text { Component } \\
\text { relation }\end{array}$ & Component A & Component B \\
\hline 1 & Q1 EP & SSQ front \\
2 & SSQ front & SSQ back \\
3 & SSQ back & Lens skimmer \\
4 & Lens skimmer & Skimmer \\
$5^{*}$ & Skimmer & BSQ front \\
6 & BSQ front & BSQ Back \\
7 & BSQ back & Skimmer 2 \\
\hline
\end{tabular}

* Component relation used by Lopez-Hilfiker et al. (2016).

highlights the need for significant characterization of collisional dissociation in the API (Aljawhary et al., 2013). The authors tune a HR-TOF-CIMS to a "weak-field mode" for iodide adduct and water cluster CIMS operation. A "strongfield mode" is used while operating in acetate mode. Comparing the negative ion mode voltage configurations under strong-field and weak-field operation presented in the supplementary information shows numerous voltage relationships that may lead to subtle differences in relative ion transmission efficiency. This problem is not unique, and authors rarely publish exact voltage configurations as the exact voltages needed to tune the API will vary across instruments. The lack of careful study when configuring the API is obvious in the available HR-TOF-CIMS literature using acetate CI where reported [acetate + acetic acid] to acetate ratios (referred to here after as the acetate cluster ratio) vary by orders of magnitude (Brophy and Farmer, 2015). For example, Bertram et al. (2011) report an acetate cluster ratio of $2.6 \times 10^{-3}$, Mohr et al. (2013) report an acetate cluster ratio of 0.07, Brophy and Farmer (2015) report an acetate cluster ratio of 0.02, and Chhabra et al. (2015) report an acetate cluster ratio of 0.2 .

We present a comprehensive characterization of the Tofwerk API. This characterization of the API allows for a detailed investigation of the acetate ionization mechanisms and the impact of controlling for these mechanisms with collisional dissociation in the ion transfer optics on sensitivity, detection limits, selectivity, and mass spectral ambiguity with the general aim of non-targeted analysis. We show that the majority, if not all, ion-neutral chemistry occurs in the ion molecule reactor (IMR) where incoming sample air mixes with the output of the ion source. Lastly, we provide insight on configuring these HR-TOF-CIMS systems for nontargeted analysis and the detection of clusters.

\section{Methods}

\subsection{Instrument description and chemical ionization source}

The HR-TOF-CIMS (Tofwerk AG and Aerodyne Research, Inc.) is described extensively in the literature (Bertram et al., 2011; Brophy and Farmer, 2015; DeCarlo et al., 2006; Jokinen et al., 2012; Junninen et al., 2010; Lee et al., 2014). The instrument described herein is operated in the negative ion mode with acetate reagent ions. The configuration is described in detail by Brophy and Farmer (2015). Notable differences include the use of a larger SH-112 single scroll pump (Agilent Technologies, Inc.) backing the IMR, a custom-built quartz glass reservoir with metal to quartz fittings for holding the reagent precursor, and the use of the standard IMR critical orifice for sampling from atmospheric pressure at $1900 \mathrm{sccm}$. Mass spectra are acquired at an extraction frequency of $25.0 \mathrm{kHz}$ and pre-averaged to $1 \mathrm{~s}$ mass spectra over a mass range from 2 to $494 \mathrm{~m} / \mathrm{z}$ using an analog to digital converter (ADQ1600 SP Devices). Instrument resolution is $>5000$ for peaks above $\sim 100 \mathrm{~m} / \mathrm{z}$. A TofDaq recorder is used to configure TOF acquisition parameters (Tofwerk AG, ToFDaq Version 1.97) and record mass spectra.

The quartz glass reagent reservoir is filled with acetic anhydride (Sigma-Aldrich, $\geq 99 \%$ ReagentPlus Grade) and the headspace is continually flushed with $\sim 10 \mathrm{sccm}$ ultrahigh purity (UHP) $\mathrm{N}_{2}$ from a cryogenic dewar (Airgas, Inc.) using a $50 \mathrm{sccm}$ mass flow controller (MKS Instruments, Inc. 1179A) and analog controller (MKS Instruments, Inc. 247D). All connections to and from the reagent reservoir are made with instrument grade $1 / 8^{\prime \prime}$ outer diameter, $0.055^{\prime \prime}$ inner diameter stainless steel instrument-grade lines (Restek, Inc.) and stainless steel Swagelok fittings and ferrules. The saturated headspace is mixed with a diluting UHP $\mathrm{N}_{2}$ flow $(\sim 2000 \mathrm{sccm})$ controlled with a stainless steel needle valve (Swagelok). Approximately $900 \mathrm{sccm}$ of the $\mathrm{N}_{2}-$ acetic anhydride mixture is subsampled through a critical orifice (O'Keefe Controls) into a Po-210 ionizer (NRD Static Controls LLC). A short, 1/4" outer diameter piece of PEEK tubing (Vici Metronics) separates and electrically isolates the stainless steel line from the Po-210 ionizer that is directly threaded into the IMR body. The glass reservoir, stainless steel lines, and Po-210 ionizer are held at $40^{\circ} \mathrm{C}$ with a PID (proportional-integral-derivative) temperature controller and heating tape (Omega Engineering, Inc.). The IMR is held at $50^{\circ} \mathrm{C}$ using the temperature controls on board the HR-TOF-CIMS. IMR and SSQ pressures are held constant at 100 and 2 mbar, respectively.

\subsection{Experimental setup}

Gas-phase standards of formic $\left(\mathrm{CH}_{2} \mathrm{O}_{2}\right)$, propionic $\left(\mathrm{C}_{3} \mathrm{H}_{6} \mathrm{O}_{2}\right)$, butyric $\left(\mathrm{C}_{4} \mathrm{H}_{8} \mathrm{O}_{2}\right)$, methacrylic $\left(\mathrm{C}_{4} \mathrm{H}_{6} \mathrm{O}_{2}\right)$, nitric 
$\left(\mathrm{HNO}_{3}\right)$, and hydrochloric acid $(\mathrm{HCl})$ are generated using permeation tubes (KIN-TEK Laboratories, Inc.) and custombuilt permeation ovens. The ovens are continually flushed with UHP $\mathrm{N}_{2}$ at a constant flow of $\sim 50 \mathrm{sccm}$ and resistively headed to a constant temperature using PID temperature controllers (Omega Engineering, Inc.). The permeation rate of each species is determined by monitoring the mass loss of the permeation tubes over the course of months. Ultra zero grade air (Airgas Inc.) is mixed with the output of the permeation tubes to create single-component alkanoic acid standards in clean air. The same source of ultra zero grade air is also used for instrument zeros and humidified air. Humidification of zero air is accomplished by passing the air through a series of large-volume glass, custom-built water bubblers filled with LCMS grade water (Sigma-Aldrich). The humidity system operates at a constant flow and varies the relative humidity (RH) using two mass flow controllers (MKS Instruments, Inc. 1179A) and a PID loop controlling the RH from an inline RH sensor (Omega Engineering HX71-V1). The sum of two mass flow controllers is held constant by the PID control loop and the ratio of the flows are changed to produce a large range of humidified air $(0-90 \%)$. All additional flows are controlled with mass flow controllers (MKS Instruments, Inc. 1179A). The gas sample flow is sent to the HR-TOF-CIMS through a polychlorotrifluoroethylene 3-way solenoid valve with a $3 \mathrm{~mm}$ orifice (NResearch, Inc) positioned upstream from the humidified air system. The total humidified flow is set below the sampling flow of the HR-TOF-CIMS (2 standard $\mathrm{L} \mathrm{min}^{-1}$ ) and either zero air or a calibration mixture is subsampled through the 3-way valve to make up the remaining sample flow (Fig. S1 in the Supplement).

All components of this system are automated to allow for comprehensive calibrations of the six authentic acid standards under different instrument settings and different RH conditions. LabVIEW scripts (LabVIEW 2014 Version 14.0f1, National Instruments, Inc.) control the gas flows using predetermined sets of flow rates, humidity settings, and instrument voltage configurations. Multiple data acquisition devices (Labjack Inc, U12) are implemented to record all flows, RH sensor output, and valve states. The HR-TOFCIMS is controlled using the Tofwerk Application Programming Interface (Tofwerk AG, Version 1.97) from within the LabVIEW environment. All data streams read by the data acquisition devices are logged to the Tofwerk HDF files along with the HR-TOF-CIMS data.

Two general modes of operation exist for this experimental setup: full calibration mode and voltage scanning mode. Briefly, operating in full calibration mode produces one background-subtracted multipoint calibration curve at each specified RH setting. Next, LabVIEW changes the instrument voltage settings and repeats the experiment. One file is created for each instrument zero and calibration step in order to simplify data processing by averaging entire files of a fixed length. Voltage scanning mode utilizes the same flow system but maintains all the flows while switching instrument voltages. Again, a separate data file is created for each voltage configuration.

\subsection{Data analysis}

Post-processing is performed in Igor Pro (WaveMetrics Inc, Version 6.3.7.2) running Tofware (Tofwerk AG, Aerodyne Research, Inc. Version 2.5.3). Tofware is used to process, fit, and then extract HR-TOF data and auxiliary data generated from the experimental setup. Once the integrated highresolution time series are extracted, scripts developed in Igor Pro process all of the experimental data to produce calibration curve summaries and statistics. TOF duty cycle corrections are made within Tofware at $\mathrm{m} / \mathrm{z} 59$ for all data collected (Drewnick et al., 2005). Mass calibration is conducted using a three-parameter fit available within Tofware using $\mathrm{O}_{2}^{-},{ }^{35} \mathrm{Cl}^{-},{ }^{37} \mathrm{Cl}^{-}, \mathrm{CHO}_{2}^{-}, \mathrm{NO}_{2}^{-}, \mathrm{C}_{2} \mathrm{H}_{3} \mathrm{O}_{2}^{-}$, and $\mathrm{NO}_{3}^{-}$as mass calibration peaks. Additionally, $\mathrm{C}_{4} \mathrm{H}_{7} \mathrm{O}_{4}^{-}$, the [acetic acid + acetate] cluster, is also included for mass calibration when operating in clustering modes where sufficient signal from this species is detected.

Calibration experiments are normalized by the ratio of the total ion signal at each calibration step relative to the total ion signal in zero air. Traditionally, normalization is conducted using the acetate reagent ion. Under declustered settings, acetate accounts for most of the total ion signal $(>80 \%)$. These calibration experiments are complicated by voltage scanning of the API. Clustered settings retain the acetate clusters, which can contribute more of the total ion signal than acetate. Thus, the use of total ion signal is appropriate to maintain consistency in normalization procedures across a large range of clustering conditions. Experiments where only zero air is used are normalized by simply dividing the individual ion signals by the total ion signal and expressing the observed signals as a fraction of total signal.

\subsection{Voltage set determination with Thuner}

Exploration of the API component relations provides additional insight to the operation of these complex instruments. Very large sets of voltages ( $>40000$ configurations) are produced using Thuner (Tofwerk AG, Version 1.9.11.0), a design of experiment optimization software produced by Tofwerk. Thuner enables the user to establish relationships between various API components and set performance targets (resolution, sensitivity, peak shape). These component relations are used to set voltages in the API and optimize voltage settings based on the performance target criteria. For example, to optimize the instrument in a clustered mode (i.e., maintaining low electric field strengths through the API), the voltage difference between the last skimmer of the SSQ and the BSQ front can be set to a small range $(0-2 \mathrm{~V})$, allowing Thuner to test the impact of tuning each region of the API on desired parameters. Increasing the voltage dif- 


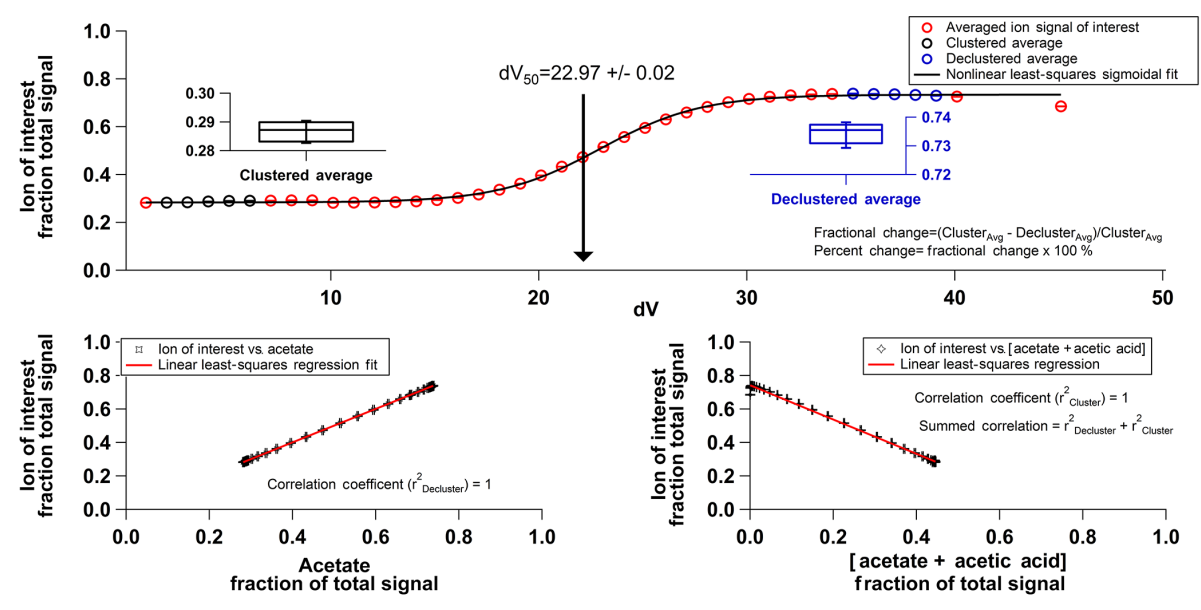

Figure 2. Top panel: an ion of interest is normalized to the total ion signal and plotted against the voltage difference for some component relation (component relation 3 is shown here). The black circles show the portion of the curve used to average the signal of the ion during operation under weak (clustered) electric field strength, with an inset box-and-whisker plot representing the clustered-average. Blue circles show the portion of the curve used to average the signal of the ion during operation under strong (declustered) electric field strength, with an inset box-and-whisker plot representing the declustered-average. The $\mathrm{dV}_{50}$ value obtained from the nonlinear least-squares sigmoidal fit is also displayed. Bottom: the correlation scatter plots of the ion of interest with acetate and the [acetic acid + acetate] cluster. Linear regression produces an $r^{2}$ correlation coefficient for the ion of interest vs. acetate (used as the model for a declustered-deprotonated species) and the ion of interest vs. [acetic acid + acetate]. These two correlation coefficients are summed and used as criteria for including or excluding declustering scans.

ference between the skimmer and the BSQ front $(2-4,4-$ $6 \mathrm{~V}$, etc.) moves the instrument stepwise from a cluster transmitting regime to a declustering regime. The SSQ and BSQ $\mathrm{RF}$ frequency and amplitude are held constant at 2.65 and $4.26 \mathrm{MHz}$ with an amplitude of 200 and $400 \mathrm{~V}$, respectively.

Maintaining instrument and sample stability is essential during these experiments, particularly when comprehensive ( $>7$ days) Thuner experiments are conducted. For this reason, the instrument samples ultra zero air throughout the entire Thuner experiment. Further details from these experiments are presented in the Supplement (Sect. S2). Ultimately, a single voltage configuration at a voltage difference of $1 \mathrm{~V}$ between the last skimmer and the BSQ front are chosen by filtering for sensitivity, resolution, and the acetate cluster ratio using the Thuner XML output and various scripts written in Igor Pro. This voltage starting point is used to create all the voltage set point files for the voltage scanning experiments (Table S1). Seven component relations are defined between adjacent components (Fig. 1 and Table 1). The component nearest the TOF is held constant, while all components upstream are floated together including the IMR body itself.

\subsection{Voltage scanning and cluster detection}

We use nonlinear least-squares sigmoidal regression following the work by Lopez-Hilfiker et al. to describe declustering voltage scans and determine the characteristic voltage $\left(\mathrm{dV}_{50}\right)$ at half signal maximum (Lopez-Hilfiker et al., 2016). The work by Lopez-Hilfiker et al. (2016) focuses on the sensitivities for iodide ion-neutral clusters and only examines declus- tering scans of species initially clustered with iodide that fall apart upon increasing the electric field strength between two components. In contrast, acetate CIMS produces both ionneutral clusters with acetate (and other negatively charged ions) and deprotonated-declustered ions. This more complex case means that ion signals may either increase or decrease as the electric field strength increases during a voltage scan. As such, we introduce another parameter to further describe the behavior of ions detected in the high-resolution mass spectrum: positive and negative change. Figure 2 details the fitting procedure and quantification of this change. A stable region at low-voltage differences (low electric filed strength, high cluster transmission) is averaged and compared to a stable region at high-voltage differences (high electric field strength, low cluster transmission) using a Student's $t$ test. If the null hypothesis (the two populations are the same) is rejected, then the percent change is calculated. This allows for the $\mathrm{dV}_{50}$ to be converted into a positive or negative number.

Lopez-Hilfiker et al. (2016) filter their fits based on the criterion that if the mean square residual is $>10 \%$, the fit is rejected. This is insufficient for our purposes of characterizing this more complex set of voltage scans. Instead, we first reject unreasonable $\mathrm{dV}_{50}$ values where the calculated $\mathrm{dV}_{50}$ is greater than twice the scan range. Next, we use the output of the Student's $t$ test to remove voltage scans with start and end points that likely belong to the same population ( $3 \mathrm{~s}$ certainty). Lastly, linear least-squares cross correlation is performed on the high-resolution time series to identify correlation with the acetate reagent ion and the [ac- 

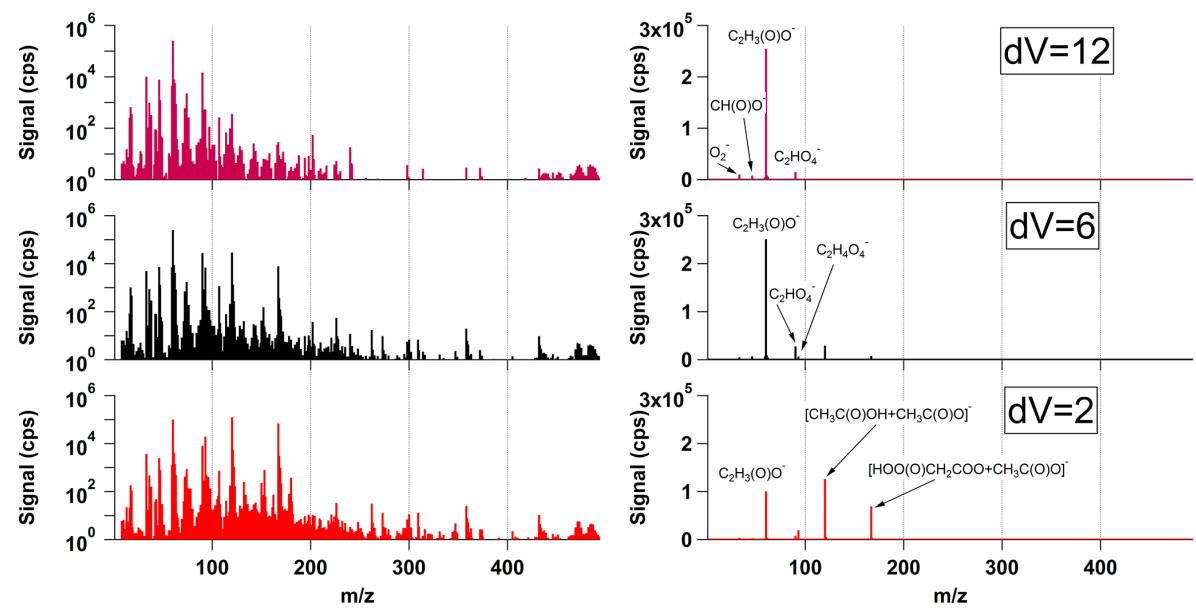

Figure 3. A representative background mass spectrum obtained by overflowing the IMR with zero air is shown at three voltage differences (component relation 5). Both the log-scale mass spectrum (left column) and linear-scale (right column) mass spectrum are displayed. Dominant peaks related to the reagent ion chemistry are labeled.

etate + acetic acid $]^{-}$cluster. The acetate reagent ion follows an increasing sigmoidal shape due to the declustering of various acetate (or other anion)-containing clusters and thus acts as a model shape for deprotonated-declustered species. The opposite is true for the [acetate + acetic acid] ${ }^{-}$cluster: the decluster scan follows a decreasing sigmoidal shape characteristic of clusters breaking apart. The sum of the correlation coefficients of the species of interest vs. acetate and the species of interest vs. the $\left[\right.$ acetate + acetic acid] ${ }^{-}$provides a final cutoff $\left(r_{\text {sum }}^{2} \geq 1.5\right)$. Most sigmoidal fits that remain follow anticipated declustering or clustering shapes.

\section{Results}

\subsection{Typical mass spectrum and voltage scanning}

The overall effect of voltage scanning on the observed mass spectrum using acetate CIMS is partially described in previous work characterizing the application of the Tofwerk API with a C-TOF-CIMS (Bertram et al., 2011). Our use of a HRTOF-CIMS enables further identification of dominant peaks in the mass spectrum and a more comprehensive analysis of tuning effects and ionization chemistry. Figure 3 shows both the log-scale mass spectrum and linear-scale mass spectrum collected while flowing ultra zero air into the inlet and changing the voltage difference between the skimmer and BSQ front (component relation 5). The mass spectrum collected under high electric field strength $(\mathrm{dV}=12)$ is dominated by the acetate reagent ion $(m / z 59.014,80.1 \%$ total signal) with a small contribution from $\mathrm{O}_{2}^{-}(3.2 \%$ total signal), $\mathrm{CHO}_{2}^{-}$(2.4\% total signal), and $\mathrm{C}_{2} \mathrm{HO}_{4}^{-}$(4.7\% total signal). Decreasing the voltage difference by half $(\mathrm{dV}=6)$ decreases the acetate reagent ion contribution $(67.8 \%$ total signal) while enabling the appearance of the [acetic acid + acetate $]^{-}$cluster $(m / z 119.035,7.9 \%$ total signal $)$ and contributing a small amount of $\mathrm{C}_{2} \mathrm{H}_{4} \mathrm{O}_{4}^{-}(1.85 \%$ total signal) and [acetate $\left.+\mathrm{C}_{2} \mathrm{H}_{3} \mathrm{O}_{5}\right]^{-}(\mathrm{m} / \mathrm{z} \quad 166.012,2.1 \%$ total signal). The most clustered settings $(\mathrm{dV}=2)$ completely change the reagent ion distribution. The acetate reagent ion decreases significantly (28\% total signal) and both the [acetic acid + acetate] ${ }^{-}$cluster and [acetate $\left.+\mathrm{C}_{2} \mathrm{H}_{3} \mathrm{O}_{5}\right]^{-}$ cluster increase drastically (35.2 and $19.3 \%$, respectively).

The appearance of $\mathrm{C}_{2} \mathrm{H}_{3} \mathrm{O}_{5}$ clustered with acetate is unanticipated. We suggest this cluster is a radical fragment from the reagent precursor (acetic anhydride) that rapidly undergoes auto-oxidation with two $\mathrm{O}_{2}$ molecules (Sect. S3). Overflowing the IMR with UHP $\mathrm{N}_{2}$ and eliminating $\mathrm{O}_{2}$ completely removes this observed cluster. Bertram et al. (2011) observe even more clustering than herein, with the higher order cluster [(acetic acid $)_{2}+$ acetate $]^{-}$nearly equal to the acetate ion. Another interesting feature of the mass spectrum presented by Bertram et al. is the presence of a peak at $m / z 166$, potentially corresponding to the [acetate $\left.+\mathrm{C}_{2} \mathrm{H}_{3} \mathrm{O}_{5}\right]^{-}$cluster. Despite observing a large abundance of the $\left[(\text { acetic acid })_{2}+\text { acetate }\right]^{-}$cluster, [acetate $\left.+\mathrm{C}_{2} \mathrm{H}_{3} \mathrm{O}_{5}\right]^{-}$remains quite small and may point to other operational differences such as the amount of acetic anhydride added to the Po-210 ionizer. The effect of the amount of acetic anhydride remains an unanswered question in the literature, but we present preliminary experiments addressing this variable in the Supplement (Sect. S4).

\subsection{API component relations}

Voltage scans and cluster control have been discussed in terms of the voltage difference between the skimmer and the BSQ front (component relation 5), but numerous other component relations exist that may be suitable for controlling collisional dissociation. To address other component re- 

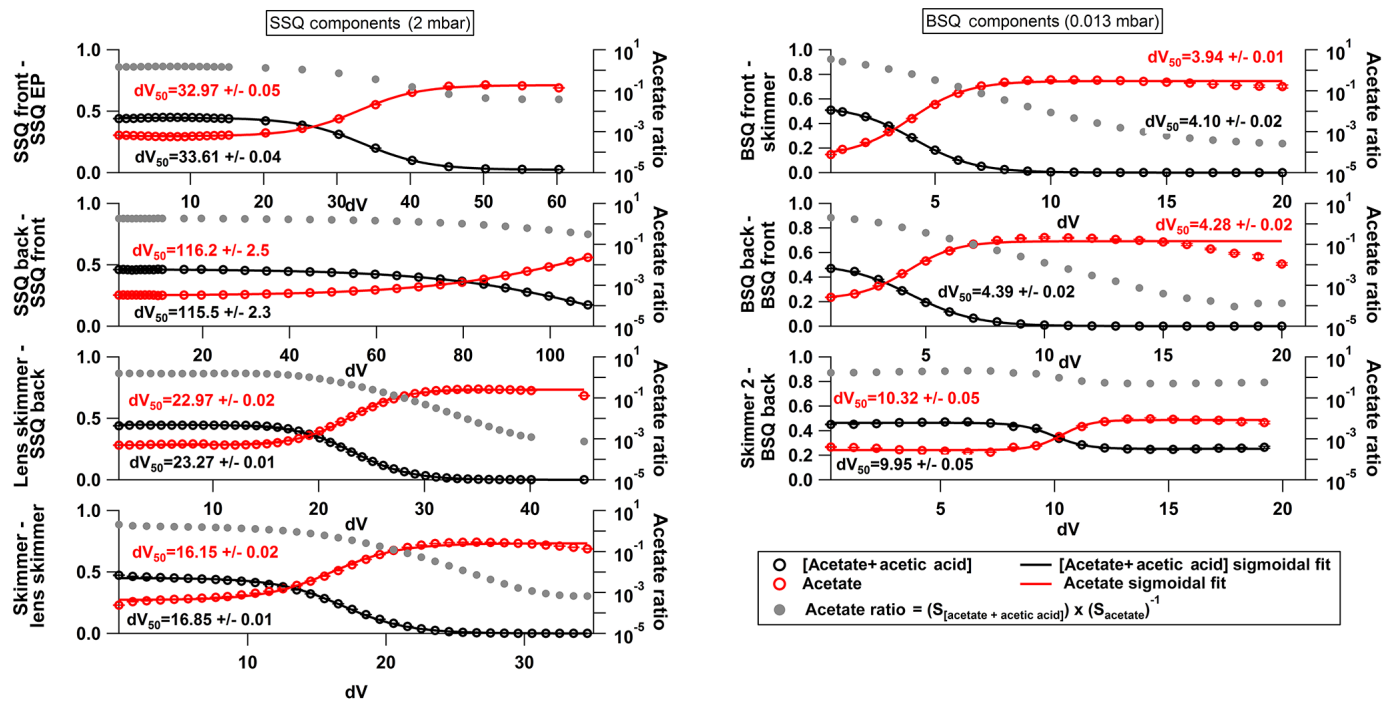

Figure 4. Voltage scan results conducted between the seven component relations in the API. Acetate (red dots) and the [acetic acid + acetate] cluster (black dots) are plotted on the left axis. Fitted $\mathrm{dV}_{50}$ values for acetate (red trace) and the [acetic acid + acetate] cluster (black trace) are included along with the least-squares sigmoidal fit. The acetate ratio (gray dots) is plotted on the right axis as a log scale. Left column: components housed in the vacuum chamber containing the SSQ ( 2 mbar). Right column: components housed in the vacuum chamber containing the BSQ (0.013 mbar).

lations, dry ultra zero air is flowed into the instrument inlet, and acetate and the first cluster, [acetic acid + acetate $^{-}$, are examined as model compounds for deprotonated-declustered species and cluster species, respectively (see Sect. 2.5). Figure 4 shows the voltage scans for adjacent components in the API. It is apparent that while the $\mathrm{dV}_{50}$ for acetate and the [acetic acid + acetate $]^{-}$cluster is extremely similar for any given set of component voltages, the $\mathrm{dV}_{50}$ for these species varies substantially across components. This is due to differences in both component spacing and pressure in the two regions of the API (BSQ, SSQ), resulting in different electric field strengths at the same voltage difference. The [acetic acid + acetate $^{-}$to acetate ratio is also displayed in Fig. 4. This ratio is reported in a number of acetate CIMS publications (discussed in the Introduction) and provides a direct comparison between instruments to describe the extent of clustering. Converting the applied voltage differences into units of Townsend (Td) (i.e., electric field strength, $E$, normalized to the number density, $N$ ) shows orders of magnitude variability in the fitted $\mathrm{dV}_{50}$ values for acetate and the [acetic acid + acetate] $]^{-}$cluster. Thus, the $E / N$ formulation fails to explain the collisional dissociation energies between various components in this system.

If the voltage difference between adjacent components is set with a voltage difference of $0 \mathrm{~V}$, ion flow through these components is controlled by fluid mechanics alone, and a decrease in ion transmission efficiency is observed. Thus, there is a lower limit to how gently one can transmit ions through these components while maintaining an electric field and high ion transmission efficiency. Deviations from the sigmoidal fit are observed at higher voltage differences for the axial voltage component on the BSQ (component relation 6). This field is applied between the BSQ back and BSQ front, but this deviation is attributable to ion transmission effects through the BSQ. This feature does not appear with the SSQ (component relation 2) because sufficiently high voltages needed to complete the curve could not be achieved due to voltage limits applied to the API to prevent electrical discharge. Another interesting feature is observed when scanning the second skimmer, located after the BSQ, and the BSQ back (component relation 7). Here, the cluster never reaches zero and the acetate signal remains correspondingly low in comparison to other components.

The exit of the SSQ to the lens skimmer (component relation 3) provides a promising region for cluster control compared to the choice of components used in previous studies (component relation 5) (Lopez-Hilfiker et al., 2016). The $\mathrm{dV}_{50}$ of the [iodide + water] cluster cannot be resolved because sufficiently weak electric fields cannot be generated. Similarly, the $\mathrm{dV}_{50}$ for the [iodide + formic acid] cluster can only be partially resolved. Scanning the BSQ front and skimmer relation (component relation 5) for the relevant acetate ions and ion-neutral clusters results in the identical problem described by Lopez-Hilfiker et al. (2016) when attempting to resolve the entire sigmoidal curve: we are unable to generate sufficiently low electric fields needed to transmit weakly bound clusters. In contrast, the exit of the SSQ to lens skimmer (component 3 ) better allows us to quantify stable cluster and decluster regions; this greatly improves our ability to detect clusters during non-targeted voltage switching experi- 


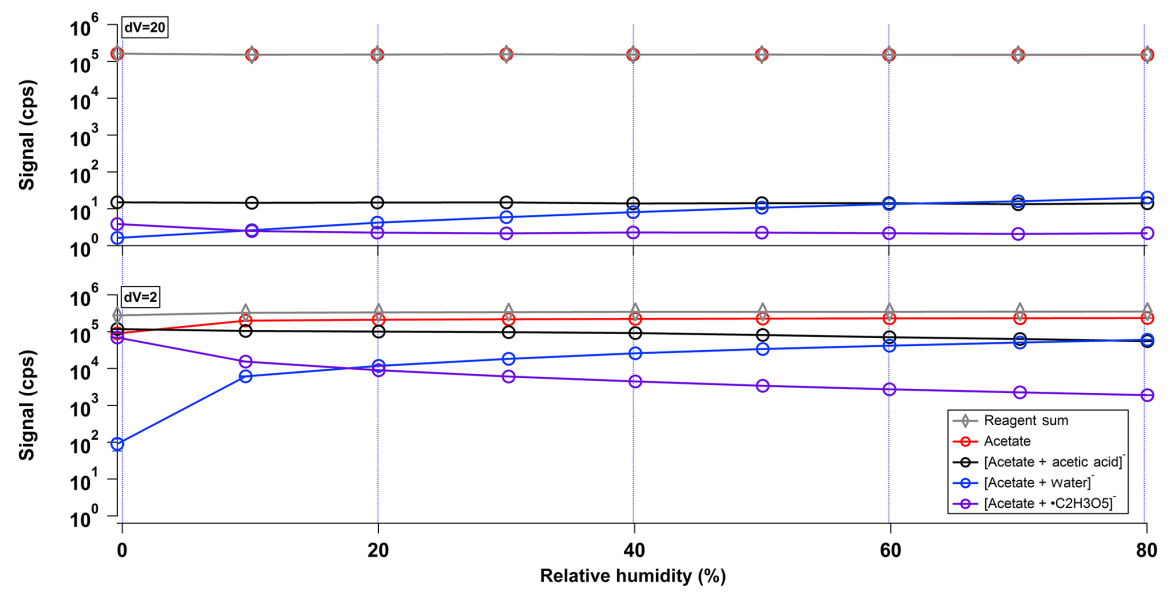

Figure 5. The effect of water vapor on various reagent ions is shown under two voltage settings, $d V=2$ (clustered) and $d V=20$ (declustered), at component relation 5 .

ments because there are stable regions that can be defined as clustered and declustered (see Sects. 2.5 and 3.6).

\subsection{Acetate CIMS ion chemistry}

Operating the HR-TOF-CIMS with acetate reagent ions in a clustering mode provides a more representative view of the ion-neutral chemistry occurring in the IMR than the declustered mode. One interesting observation is that despite the relatively high pressure in the SSQ region $(2 \mathrm{mbar})$ ionneutral clusters do not appear to form in this region. One can attribute all the ion-neutral clustering chemistry to either reactions in the IMR or cluster condensation during the jet expansion from the IMR into the SSQ. This is inferred because clustering can be controlled between the SSQ entrance plate and the SSQ front (component relation 1). After passing through this region, the ions must make it through the entire length of the SSQ and subsequent skimmers, making up most of the residence time through this region.

$\mathrm{RH}$ effects on the reagent ions are investigated while operating the HR-TOF-CIMS in both cluster mode (component relation $5, \mathrm{dV}=2$ ) and declustered mode (component relation $5, \mathrm{dV}=20$ ) (Fig. 5). Using dry ultra zero air, the abundances of acetate, [acetate + acetic acid] ${ }^{-}$cluster, and [acetate $\left.+\mathrm{C}_{2} \mathrm{H}_{3} \mathrm{O}_{5}\right]^{-}$radical cluster are quite similar. Upon the addition of water, these abundances drastically change with the appearance of an [acetate + water] $]^{-}$cluster. The [acetate + water $^{-}{ }^{-}$cluster competes with the other clusters while the sum total of acetate, [acetate + acetic acid] ${ }^{-}$, [acetate $\left.+\mathrm{C}_{2} \mathrm{H}_{3} \mathrm{O}_{5}\right]^{-}$, and [acetate + water $]^{-}$remains unchanged. We note that the [acetate + water $]^{-}$cluster is observed to increase under the highly declustered settings (component relation $5, \mathrm{dV}=20$ ), although it only makes up a small fraction of the total signal $(\sim 0.01 \%$ total ion signal $)$ compared to the clustered settings ( $\sim 17 \%$ total ion signal) at the highest RH $(80 \% \mathrm{RH})$.
Although Veres et al. (2008) note that a collisional dissociation chamber is important to "dissociate weakly bound cluster ions such as $\mathrm{CH}_{3} \mathrm{C}(\mathrm{O}) \mathrm{O}^{-}\left(\mathrm{H}_{2} \mathrm{O}\right)_{n}$ ", neither the [acetate $\left.+\mathrm{C}_{2} \mathrm{H}_{3} \mathrm{O}_{5}\right]^{-}$nor [acetate + water] ${ }^{-}$clusters have been directly identified in previous studies. This may be due to most acetate CIMS experiments being run under relatively declustered settings. The large abundance of acetate clusters observed in this study suggests that instead of a single reagent ion, chemical ionization in acetate CIMS is controlled by a distribution of reagent ion-neutral clusters that vary with $\mathrm{RH}$ and the concentration of the acetic anhydride precursor. This is an observation consistent with Bertram et al. (2011), in which their comparison between the observed mass spectra under low and high electric field strengths leads to the realization that numerous acetate clusters exist and are involved in ionizing reactions.

\subsection{Comprehensive calibrations}

Calibrations of six acid standards exhibit similar RH and voltage dependences for both the deprotonated-declustered ions and ion-neutral clusters (Fig. 6, Sect. S5). All voltage scans are conducted between the skimmer and the BSQ front (component relation 5). Propionic acid is exemplary of the behavior of the carboxylic acids. In cluster mode, the dominant ions are the deprotonated-declustered conjugate base of the acid $\left(R^{-}\right)$, the acetate cluster [acetate $\left.+\mathrm{R}-\mathrm{H}\right]^{-}$, the water cluster $\left[\mathrm{H}_{2} \mathrm{O}+\mathrm{R}-\mathrm{H}\right]^{-}$, and the self-cluster $[\mathrm{R}-\mathrm{H}+\mathrm{R}]^{-}$. The self-cluster is not observed for methacrylic acid (Sect. S5, Fig. S9). The observation of all these clusters is suppressed by operating the instrument in a declustered mode. We reiterate that while the detection of these clusters can be eliminated in the ion transfer optics, the cluster chemistry is still occurring in the IMR. Operation in clustered mode produces linear calibration curves for all species presented. Here, we define the sensitivity as the analytical sensitivity of the in - 


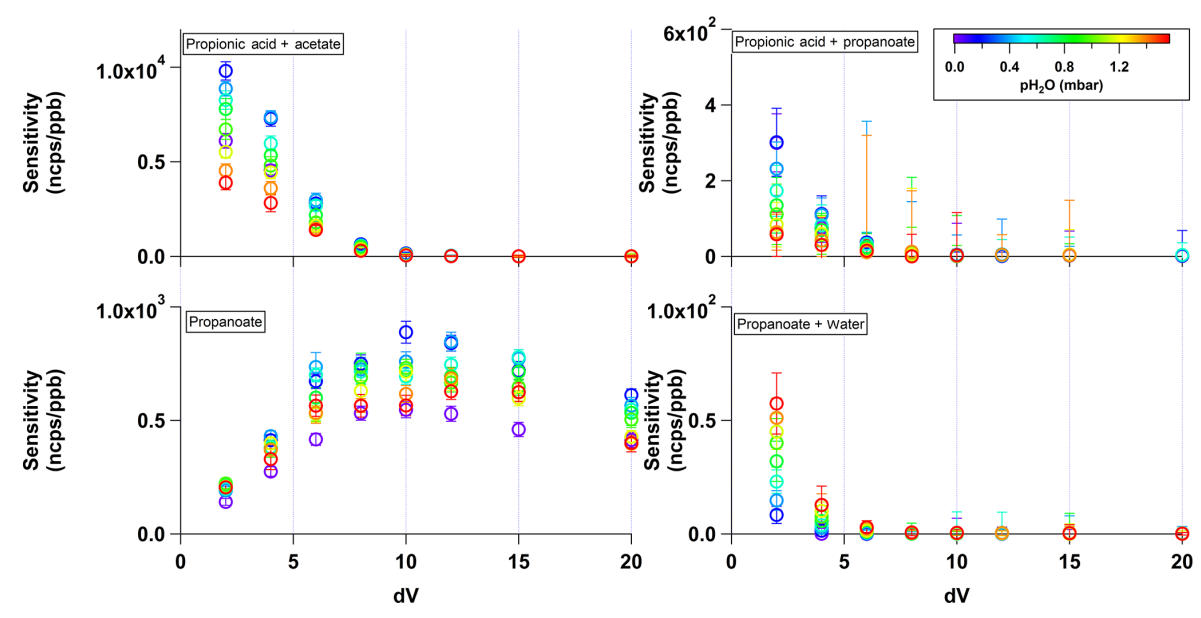

Figure 6. The sensitivity to propionic acid and related clusters is plotted against the voltage difference applied between the skimmer and BSQ front (component relation 5) in units of normalized counts per second per ppb (ncps ppb $\left.{ }^{-1}\right)$. The points are colored by the calculated water vapor content in the IMR corresponding to changing the relative humidity from 0 to $80 \%$ under laboratory conditions.

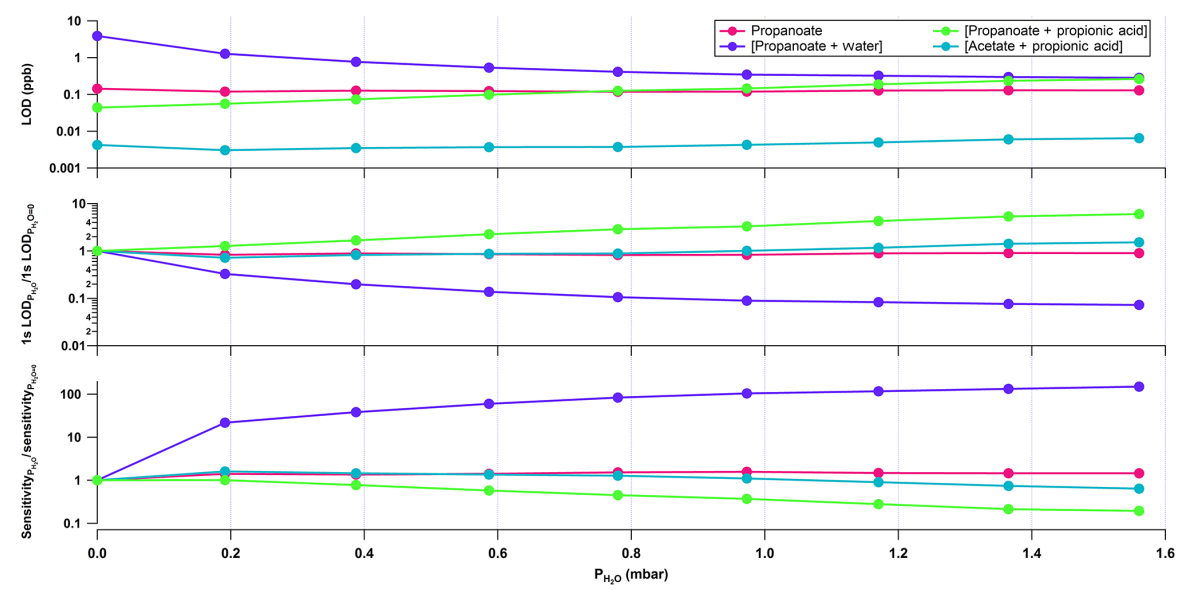

Figure 7. The propionic acid data used in Fig. 6 at a dV $=2$ are replotted as a function of water vapor content in the IMR. Top: the calculated values for the limit of detection for propionic-acid-related ions $(S / N=3,1 \mathrm{~s}$ integration). Middle: the limit of detection $(S / N=3,1 \mathrm{~s}$ integration) relative to the limit of detection under dry conditions. Bottom: the change in sensitivity relative to the sensitivity under dry conditions.

strument; the sensitivity is the observed response to a known concentration, which is a function of both ion chemistry and instrument operational parameters. In clustered mode, the most sensitive ion for the four carboxylic acids is the acetate cluster, although the sensitivities for this cluster rapidly decrease as the electric field strength is increased and the clusters are broken up. Increasing the electric field strength simultaneously increases the calculated sensitivity of all the acids at their deprotonated-declustered mass to a point where either ion transmission effects or fragmentation begin to occur and lower the sensitivity. The most sensitive ion for the two inorganic acids in cluster mode (hydrochloric acid and nitric acid) is the deprotonated-declustered ion. The [nitrate + nitric acid] $]^{-}$self cluster also shows high sensitivity for nitric acid.
The RH dependence of these clusters proceeds in the same manner as the reagent clusters. Increasing water vapor concentration in the IMR (or RH in the sample line) decreases the [acetate $+\mathrm{R}-\mathrm{H}]^{-}$cluster by forming the associated water cluster. Thus, the water cluster and acetate cluster have opposite RH effects. The same effect is observed for the self-cluster. The deprotonated-declustered ion is more difficult to reconcile. Under dry conditions, the deprotonateddeclustered ion exhibits the lowest sensitivity. Increasing the water vapor content leads to an initial increase in sensitivity, followed by a suppression of sensitivity at the highest water vapor content.

These effects can be clearly observed by examining a single voltage set corresponding to a vertical slice of Fig. 6. Figure 7 shows this picture at $\mathrm{dV}=2$ and examines the change 


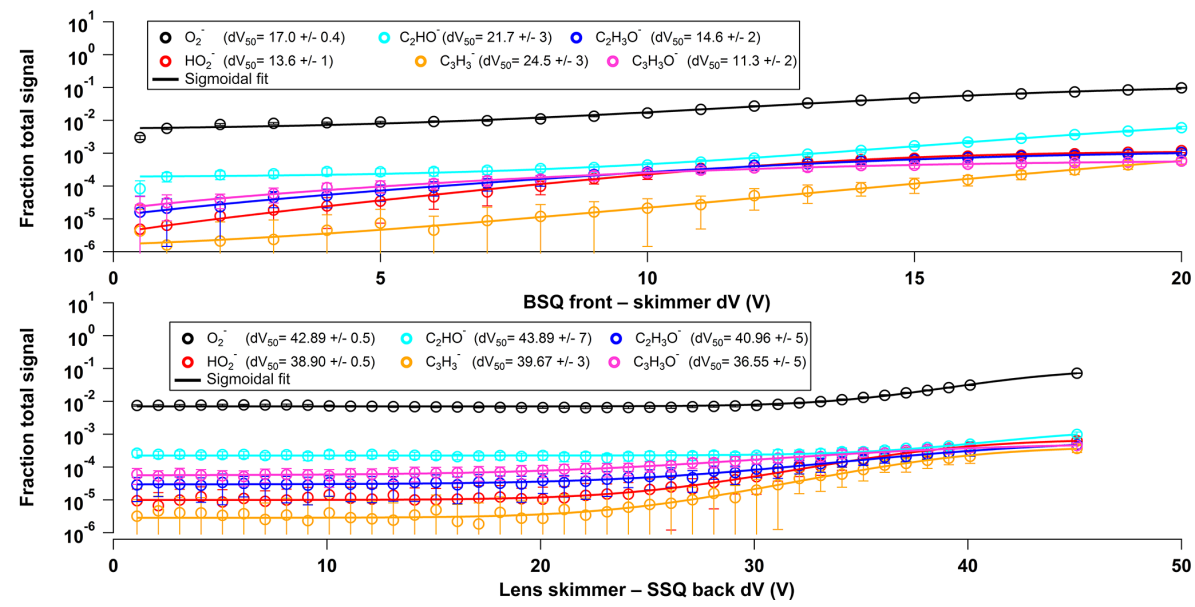

Figure 8. Voltage scanning experiments for a variety of potential fragment ions. Top: component relation 5. Bottom: component relation 3.

in sensitivity as a function of IMR water content. Here, the sensitivity to water vapor is normalized to the sensitivity under dry conditions following the work by Lee et al. (2014). The change in sensitivity to the [propanoate $+\mathrm{H}_{2} \mathrm{O}$ ] ${ }^{-}$cluster increases 149-fold while the sensitivity of the self-cluster and acetate cluster decrease by a factor of 5 and 1.6, respectively. For all analyte acids, the sensitivity of the deprotonateddeclustered ions changes by, at most, a factor of 2 as a function of water vapor concentration, indicating a robust signal.

Acetate CIMS measurements are characterized by high background count rates which affect the limit of detection (LOD). The LOD is calculated for all calibration curves (Sect. S5.3). The LODs of propionic acid ions detected in cluster mode are plotted in Fig. 7. The high sensitivity to the [acetate $+\mathrm{R}-\mathrm{H}]^{-}$clusters leads to a very low $1 \mathrm{~s} \operatorname{LOD}(S / N=3)$ below $10 \mathrm{ppt}$ for the alkanoic acids detected as clusters. The same acids have higher LODs operating in declustered mode $\sim 50-100 \mathrm{ppt}$, examining the deprotonated-declustered ions. The lower sensitivity towards the inorganic acids produces much higher LODs. Deprotonated-declustered nitric acid and hydrochloric acid have $1 \mathrm{~s}$ LODs $(S / N=3)$ of $2-4 \mathrm{ppb}$ and $<8 \mathrm{ppb}$, respectively.

These low LODs for the [acetate $+\mathrm{R}-\mathrm{H}]^{-}$carboxylic acid clusters are attributable to the lower background count rates observed at higher $m / z$ and the higher sensitivity of the [acetate $+\mathrm{R}-\mathrm{H}]^{-}$carboxylic acid clusters. Increasing the voltage difference decreases the sensitivity to these clusters; therefore we calculate very high LODs. Under normal field operating conditions, these clusters will not contribute substantially to the observed mass spectrum. This greatly improves our ability to identify and extract molecular information by decreasing mass spectral complexity; artifacts like water clusters and self- or cross-clustering reactions are eliminated. The Supplement (Sect. S5.3) provides a more detailed presentation of the LODs for all calibrated species.

\subsection{Evidence of fragmentation}

Molecular fragmentation can occur at high electric field strengths. Specific ions observed in the mass spectrum enable investigation of the voltages at which fragmentation onsets. We investigate fragmentation between the SSQ back and the lens skimmer (component relation 3) and between the skimmer and BSQ front (component relation 5). We identify at least six ions $\left(\mathrm{O}_{2}^{-}, \mathrm{C}_{2} \mathrm{HO}^{-}, \mathrm{C}_{2} \mathrm{H}_{3} \mathrm{O}^{-}, \mathrm{HO}_{2}^{-}, \mathrm{C}_{3} \mathrm{H}_{3}^{-}\right.$, $\mathrm{C}_{3} \mathrm{H}_{3} \mathrm{O}^{-}$) with very high $\mathrm{dV}_{50}$ values and molecular formulae consistent with fragmentation (Fig. 8). Fragmentation must be considered when configuring a CIMS experiment to avoid destroying compounds of interest. Scanning component relation 3 exhibits a clear fragmentation curve, with most $\mathrm{dV}_{50}$ values occurring at $\mathrm{dV}=40$. This is about twice as large as the $\mathrm{dV}_{50}$ values observed for the [acetate + acetic acid] ${ }^{-}$cluster. The sigmoidal fits for component relation 5 are less obvious, but calculated $\mathrm{dV}_{50}$ values for the fragment ions are nearly 4 times as large as observed for the [acetate + acetic acid] $]^{-}$cluster. The possibility of molecular fragmentation may explain some of the observed decreases in sensitivity at the higher $\mathrm{dV}$ values for the deprotonateddeclustered ions (Fig. 6). It remains challenging to separate the fragmentation effect from ion transmission effects that may dominate under very high electric field strengths (dV $>40 \mathrm{~V}$ component relation $3, \mathrm{dV}>15 \mathrm{~V}$ component relation 5).

\subsection{Complex sample declustering and implications for ambient atmospheric data}

We use a potential aerosol mass (PAM) chamber to create a complex mixture of oxidized organic species in high concentrations from the oxidation of $\alpha$-pinene by $\mathrm{OH}$. The purpose of this experiment is not to make any claims about $\alpha$ pinene $+\mathrm{OH}$ chemistry but instead to generate a complex mixture of oxygenated organic molecules that is not currently 

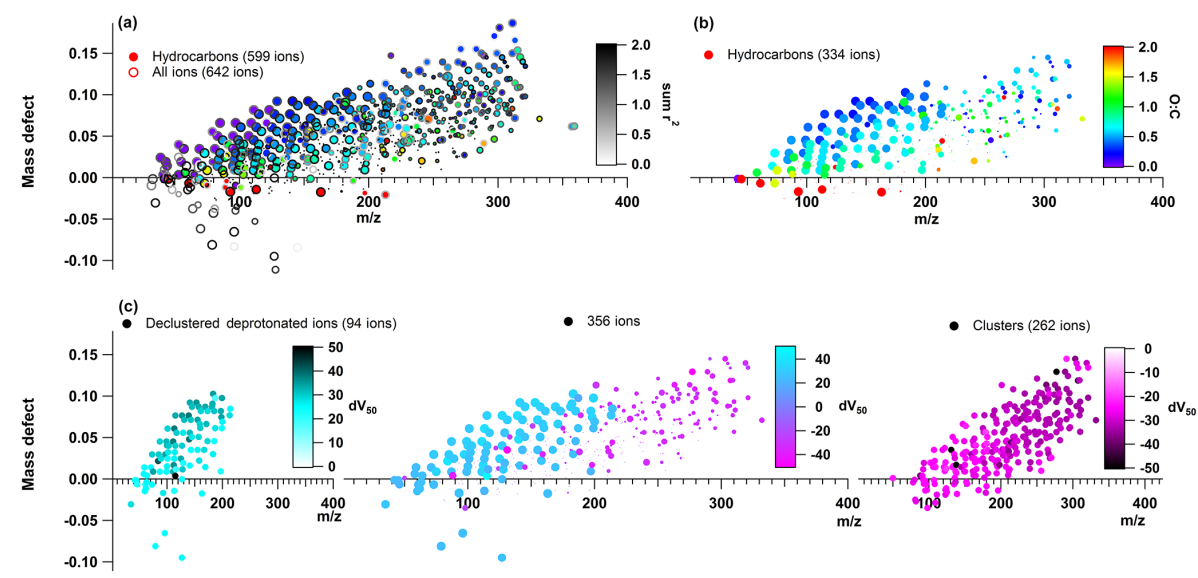

Figure 9. Mass defect plots from scanning component relation 3 during the $\alpha$-pinene PAM chamber experiment. (a) All species (642 ions) are plotted as open circles and colored by the sum correlation coefficient (gray color-scale). Hydrocarbons (599 ions) are plotted as solid circles and colored by the calculated oxygen to carbon ratio (rainbow color scale). Both the open circles and solid circles are sized by the percent change during the scanning experiment. Small circles are decreasing as a function of increasing electric field strength while large circles are increasing. (b) Hydrocarbons that meet the scanning criteria (334 ions) are plotted as solid circles colored by their oxygen to carbon ratio (rainbow color-scale); these are also sized by the percent change during the scanning experiment. (c) Left: ions ( 94 non-clusters) which meet the scanning criteria and increase during a voltage scan $\left(+\mathrm{dV}_{50}\right)$ are plotted as solid circles (Cyan color scale). Middle: ions which meet the scanning criteria (334 ions) are colored by $\mathrm{dV}_{50}$ (cyan-magenta color-scale). Right: ions (262 clusters) which meet the scanning criteria and decrease during a voltage scan $\left(-\mathrm{dV}_{50}\right)$ are plotted as solid circles (magenta color scale).
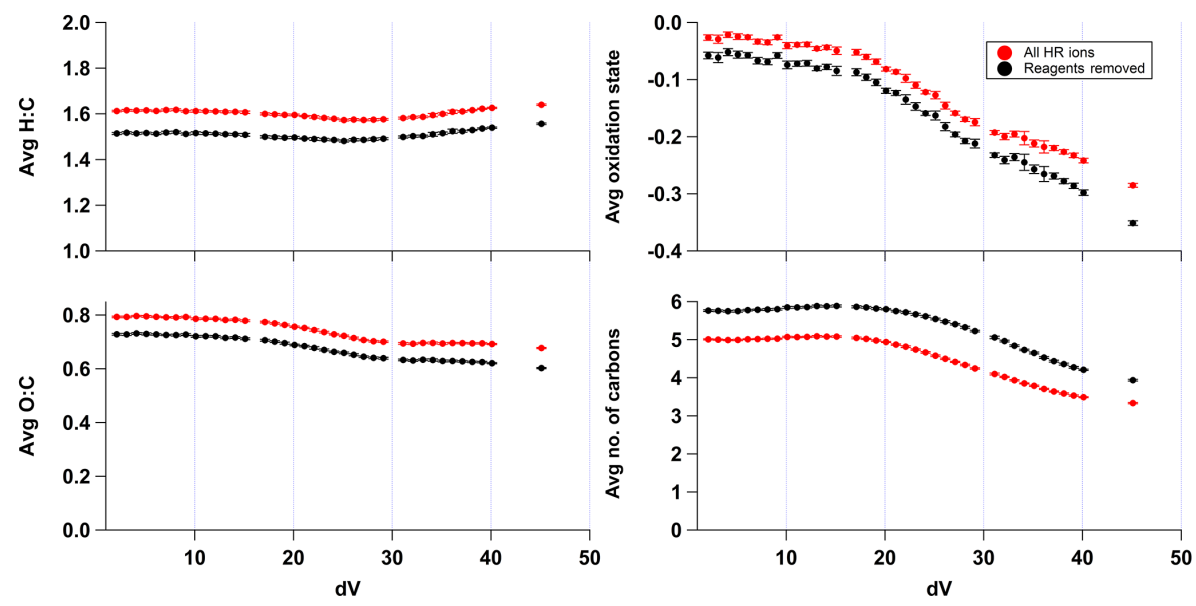

Figure 10. Bulk properties calculated in Tofware during the $\alpha$-pinene PAM chamber experiment are plotted as a function of dV (component relation 3).

possible to obtain through authentic standards. The configuration of the PAM chamber is described in detail by Friedman et al. (2016). Briefly, a flow of $\sim 65 \mathrm{ppbv} \alpha$-pinene is oxidized by $2.8 \times 10^{6}$ molecules $\mathrm{cm}^{-3} \mathrm{OH}$ (or $\sim 1.9$ days equivalent $\mathrm{OH}$ exposure) in a $13.1 \mathrm{~L}$ PAM chamber in $\sim 2 \mathrm{~min}$. Declustering scans between the SSQ back and lens skimmer (component relation 3) changes the observed mass spectrum and is best observed in mass defect space (Fig. 9). The $\mathrm{dV}_{50}$ values obtained for the clustered ions show an increasing trend with increasing mass, consistent with Lopez-Hilifiker et al. (2016)'s observation that large multifunctional nitrates and large oxygenated species exhibit high $\mathrm{dV}_{50}$ values.
Bulk descriptive values are calculated by Tofware using the ion signal intensity to weight the contribution of each individual ion to the total signal (Fig. 10). This approach is frequently conducted with the HR-TOF-CIMS, either without correcting for differences in sensitivity (Friedman et al., 2016), or by applying the sensitivity of one species (typically formic acid) to every species (Chhabra et al., 2015). The main finding is that the average oxygen to carbon ratio $(\mathrm{O}: \mathrm{C})$, hydrogen to carbon ratio $(\mathrm{H}: \mathrm{C})$, oxidation state, and carbon number (number of carbons) all change significantly as a function of applied voltage difference. The average number of carbons per ion decreases by $\sim 1.7$ car- 
Table 2. Alkanoic acid species scanning results during authentic standard API scanning (left column) and PAM chamber scanning (right column). $\mathrm{dV}_{50}$ values are reported for the deprotonated-declustered ions (increasing signal with increasing $\mathrm{dV}$ ) and for the acetate-clustered ions (decreasing signal with increasing $\mathrm{dV}$ ).

\begin{tabular}{lrr|rr}
\hline & \multicolumn{2}{c|}{ Standard scans $\left(\mathrm{dV}_{50}\right)$} & \multicolumn{2}{c}{ PAM scans $\left(\mathrm{dV}_{50}\right)$} \\
\cline { 2 - 5 } Species & $\begin{array}{r}\text { Primary ions } \\
\text { (appearance) }\end{array}$ & $\begin{array}{r}\text { Acetate cluster } \\
\text { (disappearance) }\end{array}$ & $\begin{array}{r}\text { Primary ion } \\
\text { (appearance) }\end{array}$ & $\begin{array}{r}\text { Acetate cluster } \\
\text { (disappearance) }\end{array}$ \\
\hline Formic acid $(\mathrm{CH}(\mathrm{O}) \mathrm{OH})$ & $23.02 \pm 0.9$ & $22.62 \pm 0.4$ & $24.00 \pm 0.1$ & $22.86 \pm 0.03$ \\
Propionic acid $\left(\mathrm{C}_{2} \mathrm{H}_{5} \mathrm{C}(\mathrm{O}) \mathrm{OH}\right)$ & $24.56 \pm 0.2$ & $25.02 \pm 0.9$ & $30.15 \pm 0.8$ & $25.37 \pm 0.7$ \\
Butyric acid $\left(\mathrm{C}_{3} \mathrm{H}_{7} \mathrm{C}(\mathrm{O}) \mathrm{OH}\right)$ & $25.65 \pm 0.2$ & $26.09 \pm 0.1$ & $29.67 \pm 0.6$ & $25.81 \pm 0.3$ \\
Pentanoic acid $\left(\mathrm{C}_{4} \mathrm{H}_{9} \mathrm{C}(\mathrm{O}) \mathrm{OH}\right)$ & $\mathrm{NA}$ & $\mathrm{NA}$ & $30.15 \pm 0.8$ & $25.37 \pm 0.71$ \\
Hexanoic acid $\left(\mathrm{C}_{5} \mathrm{H}_{11} \mathrm{C}(\mathrm{O}) \mathrm{OH}\right)$ & $\mathrm{NA}$ & $\mathrm{NA}$ & $33.27 \pm 3.3$ & $(23.97 \pm 1.0)^{1}$ \\
Acetate reagent $\left(\mathrm{CH}_{3} \mathrm{C}(\mathrm{O}) \mathrm{OH}\right)$ & $23.87 \pm 0.03$ & $24.05 \pm 0.02$ & $24.03 \pm 0.05$ & $24.25 \pm 0.03$ \\
\hline
\end{tabular}
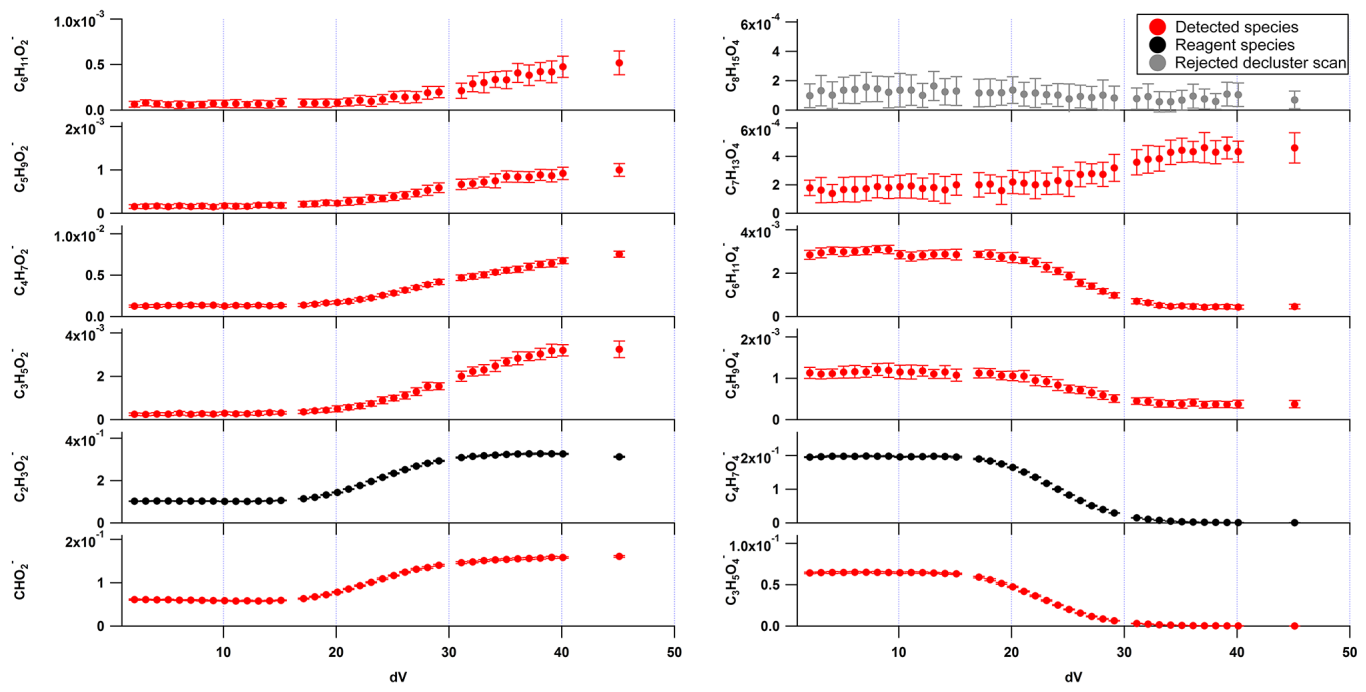

Figure 11. Individual alkanoic acid scans obtained during the scanning PAM chamber experiment (left) and [alkanoic acid + acetate] cluster scans (right).

bon atoms per ion at the most declustered voltages. This is consistent with primarily removing or declustering acetatecontaining clusters; other species are simultaneously declustered, but acetate is the most abundant ion and thus comprises the dominant cluster. The average oxidation state decreases with declustering voltage with three distinct regions: low (0$18 \mathrm{~V})$, intermediate $(18-30 \mathrm{~V})$, and high $(>30 \mathrm{~V}) \mathrm{dV}$ values. Little declustering is observed at low $\mathrm{dV}$. The observed average oxidation state remains stable and is consistent with the behavior of other bulk metrics. Acetate clusters and other ion-neutral clusters dissociate in the intermediate $\mathrm{dV}$ range, causing the steep change observed in average oxidation state. Calculated bulk oxidation state continues to decrease at high $\mathrm{dV}$ values and is either due to the onset of fragmentation or the continued dissociation of strongly bound clusters.

$\mathrm{dV}_{50}$ values obtained by scanning component relation 3 using authentic standards are compared to the $\mathrm{dV}_{50}$ values of the same ions observed during the declustering PAM experiment (Table 2). The $\mathrm{dV}_{50}$ values determined for the dis- appearance of acetate clusters using authentic standards in zero air are similar to the $\mathrm{dV}_{50}$ values obtained during the PAM scans (Fig. 11). However, the PAM declustering scan consistently shows larger $\mathrm{dV}_{50}$ values for the deprotonateddeclustered ions of all alkanoic acids. We present two hypotheses to account for this observation as follows. (1) The complex mix of species produces clusters not only with acetate, but also with other abundant ions. This is consistent with the observation of self-clusters and clusters with background ions during the single-component comprehensive calibrations (Sect. 3.4). If these clusters are more strongly bound than the acetate-containing clusters, then the destruction of the more strongly bound clusters will continue as the voltage difference increases and lead to the observed increase in $\mathrm{dV}_{50}$ values. (2) The difference in $\mathrm{dV}_{50}$ for deprotonateddeclustered ions between the standards and PAM mixture may be the result of fragmentation of multifunctional oxygenated hydrocarbons that are decomposing to ions that are isobaric with the alkanoic acids. 
The shape of the declustered-deprotonated ions during the PAM declustering scan is different from the behavior of these species during single-component declustering scans in zero air. When individual authentic standards are added to zero air and declustering depletes the acetate-carboxylic acid cluster, the corresponding deprotonated-declustered ion ceases to change. In contrast, PAM declustering scans show a continually increasing signal for the C3-C5 alkanoic acids with declustering. The signal intensity of the C3-C5 alkanoic acids during the PAM experiment is quite low in comparison to formic acid. Thus, the amount of fragmentation or declustering from strongly bound clusters must be substantial to actually observe this effect for formic acid.

\section{Discussion: acetate CIMS}

Acetate CIMS ionizes analytes by both proton abstraction (Reaction R1) and ion-neutral clustering reactions (Reacions R2-R3). Detected ions are observed as deprotonateddeclustered ions because of the collisional dissociation that occurs during the transfer of the ions from the ion source to the mass analyzer (Reaction R4). The original development of this method by Veres et al. (2008) does not investigate the importance of clustering in the ion source due to the use of a quadrupole mass spectrometer, limited mass scan range, and a collisional dissociation chamber (CDC). The idea that acetate CIMS is selective towards carboxylic acids is true, but the two ionization pathways (clustering vs. direct proton abstraction) complicate mass spectral interpretation and efficient declustering with a CDC is necessary. Thus, the selectivity of acetate towards acids is really a function of both ion-neutral chemistry and instrument operation.

We find that the acetate CIMS reagent ions and reagent ion clusters behave similarly to the detected species in both clustering behavior and effects of API declustering. The observed clustering behavior of the reagent ions with water (Fig. 5) explains the sensitivity dependence on RH (Figs. 67 , Sect. S4). During calibration, the analyte-containing clusters are shifting in abundance as a function of water vapor concentration, leading to differences in collisional dissociation efficiency and proton-abstraction efficiency. This is inconsistent with previous quadrupole acetate CIMS experiments that indicate no humidity dependence for formic acid (Veres et al., 2008). However, the ions most susceptible to humidity effects are the ion-neutral clusters; these species are rarely detected because of the operation of the API on the HR-TOF-CIMS in a declustered mode and the use of a CDC on quadrupole instruments. As such, the cluster distribution in the Veres et al. (2008) study may be completely different than the cluster distribution observed here, making comparison between these instruments and relative humidity effects nebulous. Collisional dissociation both simplifies the observed mass spectrum and eliminates the observation of acetate-containing ion-neutral clusters. Effective collisional dissociation is the key to predominantly detecting protonabstraction reaction products and maintaining the level of selectivity desired with chemical ionization. Ambient detection of IEPOX and ISOPOOH using acetate clusters (Budisulistiorini et al., 2015) will likely suffer from severe humidity effects leading to large changes in sensitivity.

Similar humidity dependences are observed with iodide adduct CIMS (Lee et al., 2014). Acetate CIMS may be simpler because the sensitivities for the deprotonateddeclustered ions follow approximately the same trend at a given voltage configuration in the API. In contrast, species clustered with iodide exhibit different RH dependences in both magnitude and shape for iodide adduct CIMS. We observe similarly complex RH dependencies in acetate and iodide reagent ions when run in a clustering mode (Fig. 7). The observation of carboxylic acids clustering with water and other ions has been observed using quadrupole instruments with atmospheric pressure ion sources (Viidanoja et al., 1998).

The role of water on acetate CIMS chemistry remains difficult to reconcile. Propionic acid sensitivities are the lowest under dry and very wet conditions (Fig. 6), but other trends exist for other deprotonated-declustered acids (Sect. S5.1). The formation of the water clusters, acetate clusters, and selfclusters shows identical RH dependence for all the calibration compounds: the addition of water shifts the cluster distribution as water is incorporated into acetate clusters. Additionally, normalization methods described herein do not eliminate the relative humidity dependence. Normalization of acetate CIMS data remains a challenge because the information about the cluster distribution is lost when collisional dissociation is sufficiently high that we observe only declustered, deprotonated ions. Relative-humidity-dependent calibrations may be the most direct method for rigorously addressing the water interference.

Controlling for clustering reactions by operating the API on the HR-TOF-CIMS under declustered settings is obvious, but the API voltage configurations do not exist as a binary system of clustered and declustered operation, making the choice of voltages a balancing act. The data presented herein indicate that operating with an acetate cluster ratio of $\sim 1 \times 10^{-2}$ is sufficient to eliminate the contribution of all clusters with carboxylic acids directly investigated here under ambient conditions. This corresponds to a dV at component relation 5 of $\sim 15 \mathrm{~V}$ and a dV at component relation 3 of $\sim 35 \mathrm{~V}$. However, these exact voltage differences may be instrument-dependent, making the acetate cluster ratio an important operational parameter that should be reported when using acetate CIMS as suggested in previous publications from this group (Brophy and Farmer, 2015). The effect of acetate precursor and acetic anhydride concentrations on sensitivity warrants further study. Acetic anhydride is a difficult reagent precursor to work with because it is difficult to quantify the mass entering the Po-210 ionizer. It is even more difficult to constrain how efficiently acetate is produced from 
the $\alpha$-particle flux, and comparison between instruments and ion sources remains nebulous.

Acetate CIMS requires significant declustering for ambient atmospheric measurement. The sensitivity to propionic acid detected as propanoate is maximized for component relation 5 at a dV of 10-12 V (Fig. 6), although the LOD and relative contribution of each cluster vs. the deprotonateddeclustered species remains surprisingly high at these voltages (see Sect. S5 for additional compounds, LODs, and sensitivity ratios). Increasing the $\mathrm{dV}$ at component relation 5 causes the relative contribution of each cluster to drop and decreases the sensitivity of the deprotonated-declustered ions (Sect. S5.2). However, these high-voltage differences lead to the formation of potential fragment ions at low $\mathrm{m} / \mathrm{z}$ (Fig. 8). We note that the average bulk parameters $(\mathrm{O}: \mathrm{C}, \mathrm{H}: \mathrm{C}$, number of carbons, oxidation state) continually change as a function of applied voltage difference (Fig. 10). The experiments calculating bulk parameters scan component relation 5, and distinct changes appear at a $\mathrm{dV}$ of $30 \mathrm{~V}$. This may support the hypothesis that fragmentation is occurring because at $\mathrm{dV}=30 \mathrm{~V}$, the average $\mathrm{H}: \mathrm{C}$ increases while the average $\mathrm{O}: \mathrm{C}$ decreases, consistent with multifunctional oxygenate fragmentation. Increasing the voltage difference between adjacent components decreases the probability of transmitting a cluster through the API, although the clusters will be detectable at sufficiently high concentrations as shown by the calculated LODs (Sect. S5.3).

Chhabra et al. (2015) present a method to account for clustering, or adduct formation, in a study of $\alpha$-pinene and naphthalene oxidation products using a PAM chamber and the acetate HR-TOF-CIMS. However, this method underestimates the complexity of the clustering problem by assuming that clustering reactions proceed only via adducts between acetate and a non-clustered ion $(\mathrm{R}-\mathrm{H})$ forming acetate clusters, $\left[\mathrm{R}-\mathrm{H}+\right.$ acetate $^{-}$. The acetate-cluster mechanism is the most dominant clustering mechanism for the carboxylic acids studied in this work because there is a very high concentration of acetate ions relative to any other species in the IMR. Nitric acid, however, provides one exception to this hypothesis. The [nitric acid + acetate $]^{-}$cluster and deprotonated declustered nitrate ion are less sensitive (i.e., contribute less to the mass spectrum total signal) relative to the [nitrate + nitric acid] self-cluster under all voltage differences and RH conditions (Sect. S5.2, Fig. S17). Thus, this approach neglects self-clustering, water-clustering, and other cross-species clustering reactions which occur, albeit to a lesser extent than acetate clustering, in the system described herein. We evaluate the Chhabra et al. methodology for accounting for acetate clusters (Sect. S6), and find that propionic acid clustering with acetate is underestimated by a factor of 15.5-26 depending on RH. Formic acid is accurately addressed under dry conditions, but is underestimated by a factor of 15 with the addition of water. Butyric acid is underestimated by a factor of $17-26$, and methacrylic acid is underestimated by a factor of 5-12 depending on $\mathrm{RH}$. The findings described herein further emphasize the importance of accounting for RH dependences of the reagent ion and thus cluster distribution.

We note two challenges in quantifying the impact of clustering on observed bulk properties or mass. (1) The presence of self-clusters and clusters formed with other ions present in the background spectrum during single-component calibrations suggests that complex mixtures will be impacted by clustering from other species; for example, ambient formic acid may form formate ions that cluster with other carboxylic acids. In situ standard additions are one approach for identifying this problem of secondary chemical ionization. (2) $\mathrm{RH}$ changes the ratio of clustered analyte to deprotonated-declustered analyte (Fig. 7, Sect. S5.2), further complicating quantification in ambient field measurements. However, controlling cluster interference in observed mass spectra by collisional dissociation is a straightforward approach to the complexity of the acetate CIMS. Other formulations proposed in the literature may oversimplify this problem.

Quantification of complex mixtures with the acetate CIMS is a complex problem. Clustering is a key mechanism for abstracting protons from carboxylic acids. Proton-abstracted declustered ions are predominantly observed if clusters are collisionally dissociated during transmission from the IMR to the detector. This suggests that some combination of both cluster binding energy and gas-phase acidity control the extent to which the analyte species retains a charge upon declustering. The prevalence of cross-clustering reactions also demonstrates that secondary ion chemistry is occurring to an appreciable extent. The challenge of quantification when sensitivity varies by both analyte and RH may be further complicated by IMR design and ion transmission through the ion optics. With all these considerations, it is remarkable that such good agreement has been found between acetate CIMS measurements and aerosol mass spectrometer data, suggesting that despite the complexities and unknowns, the acetate CIMS captures an important fraction of the gasphase chemistry relevant to secondary organic aerosol production and evolution (Aljawhary et al., 2013; Chhabra et al., 2015; Lopez-Hilfiker et al., 2016).

\section{Conclusions}

Non-targeted analysis using HR-TOF-CIMS with no preseparation is challenging, but remains a promising technique to understand atmospherically relevant species at low $(<1 \mathrm{ppb})$ concentrations. We characterize numerous operational parameters using authentic standard calibrations that drastically improve our ability to understand and interpret the acetate CIMS mass spectrum. Tuning the HR-TOF-CIMS to a declustered mode in which the acetate ratio is $\leq 1 \times 10^{-2}$ eliminates the clusters formed in the IMR. Further, we investigate the efficiency of declustering by applying the voltage 
scanning tools described herein to a complex mixture of $\alpha$ pinene oxidation products. These tools provide a convenient approach to identify whether alkanoic acid signals, for example, are solely due to the organic acid, or also the product of clustering or fragmentation reactions.

Iodide adduct and nitrate adduct CIMS may also benefit from routinely operating in a voltage scanning mode for nontargeted analysis. The iodide CIMS mass spectrum contains a poorly understood region that is separated in mass defect space from the iodide-cluster region by the "iodide valley" (Lee et al., 2014). This region is thought to contain peroxy acids $(\mathrm{R}-\mathrm{C}(\mathrm{O}) \mathrm{OOH})$ which appear as carboxylic acids upon increasing the applied voltage difference in the API. Thus, under normal iodide adduct CIMS operation, species in this region will exist as a complex mixture of ion-neutral clusters without iodide. Upon declustering, the iodide adducts will fall apart along with any of the non-iodide-containing ion-neutral clusters observed in the more positive mass defect region. This would provide an additional set of information that can be compared to the results obtained in a clustered mode where only the iodide-containing clusters are evaluated. Lastly, if the lessons learned here about acetate CIMS apply to deprotonated-declustered anions in general, one may decrease the RH dependence observed with the iodide adducts by operating in a declustered mode and examining declustered species.

The API characterization presented herein may impact the analysis of atmospheric ions and new-particle formation under both ambient and laboratory conditions, such as the Cosmics Leaving OUtdoor Droplets (CLOUD) facility. Recent publications detailing CLOUD chamber measurements show stable clusters containing up to 17 sulfuric acid molecules clustered with other species (Schobesberger et al., 2015). The authors note that water is absent from most observed clusters due to evaporation inside the API-TOF, and that other species may also fragment (Olenius et al., 2013). The literature surrounding the API-TOF further acknowledges that declustering inside the instrument is poorly understood, and that fragmentation is highly related to instrument settings (Ehn et al., 2011; Junninen et al., 2010; Olenius et al., 2013). The scanning procedures presented herein may be of particular use to API-TOF instruments, in determining both the strength of these clusters and the API control/bias on observed cluster size and composition.

The observed mass spectrum acquired using the acetate CIMS is the combined result of CI occurring in the IMR and declustering occurring throughout the instrument. Ignoring clustering will result in either an over- or underestimation of the average $\mathrm{H}: \mathrm{C}$ ratio, $\mathrm{O}: \mathrm{C}$ ratio, average oxidation state, and average number of carbons depending on the extent of clustering. Clustering is efficiently controlled using API component relations, and clusters can be identified using nonlinear least-squares sigmoidal regression and $\mathrm{dV}_{50}$ detection. The techniques and considerations described herein will be relevant for a wide variety of API-TOF users.

\section{Data availability}

Data used to prepare this paper are available upon request from the corresponding author.

\section{The Supplement related to this article is available online at doi:10.5194/amt-9-3969-2016-supplement.}

Acknowledgements. We acknowledge the National Science Foundation (AGS 1240611) and the Arnold and Mabel Beckman Foundation (Young Investigator Award) for funding this work. We would also like to acknowledge Joel Kimmel and Manuel Hutterli (Tofwerk AG) for relevant discussions and technical support.

Edited by: G. Phillips

Reviewed by: two anonymous referees

\section{References}

Aljawhary, D., Lee, A. K. Y., and Abbatt, J. P. D.: High-resolution chemical ionization mass spectrometry (ToF-CIMS): application to study SOA composition and processing, Atmos. Meas. Tech., 6, 3211-3224, doi:10.5194/amt-6-3211-2013, 2013.

Bertram, T. H., Kimmel, J. R., Crisp, T. A., Ryder, O. S., Yatavelli, R. L. N., Thornton, J. A., Cubison, M. J., Gonin, M., and Worsnop, D. R.: A field-deployable, chemical ionization timeof-flight mass spectrometer, Atmos. Meas. Tech., 4, 1471-1479, doi:10.5194/amt-4-1471-2011, 2011.

Brophy, P. and Farmer, D. K.: A switchable reagent ion high resolution time-of-flight chemical ionization mass spectrometer for real-time measurement of gas phase oxidized species: characterization from the 2013 southern oxidant and aerosol study, Atmos. Meas. Tech., 8, 2945-2959, doi:10.5194/amt-8-2945-2015, 2015.

Budisulistiorini, S. H., Li, X., Bairai, S. T., Renfro, J., Liu, Y., Liu, Y. J., McKinney, K. A., Martin, S. T., McNeill, V. F., Pye, H. O. T., Nenes, A., Neff, M. E., Stone, E. A., Mueller, S., Knote, C., Shaw, S. L., Zhang, Z., Gold, A., and Surratt, J. D.: Examining the effects of anthropogenic emissions on isoprenederived secondary organic aerosol formation during the 2013 Southern Oxidant and Aerosol Study (SOAS) at the Look Rock, Tennessee ground site, Atmos. Chem. Phys., 15, 8871-8888, doi:10.5194/acp-15-8871-2015, 2015.

Chhabra, P. S., Lambe, A. T., Canagaratna, M. R., Stark, H., Jayne, J. T., Onasch, T. B., Davidovits, P., Kimmel, J. R., and Worsnop, D. R.: Application of high-resolution time-of-flight chemical ionization mass spectrometry measurements to estimate volatility distributions of a-pinene and naphthalene oxidation products, Atmos. Meas. Tech., 8, 1-18, doi:10.5194/amt-8-1-2015, 2015.

Cubison, M. J. and Jimenez, J. L.: Statistical precision of the intensities retrieved from constrained fitting of overlapping peaks in high-resolution mass spectra, Atmos. Meas. Tech., 8, 23332345, doi:10.5194/amt-8-2333-2015, 2015. 
DeCarlo, P. F., Kimmel, J. R., Trimborn, A., Northway, M. J., Jayne, J. T., Aiken, A. C., Gonin, M., Fuhrer, K., Horvath, T., Docherty, K. S., Worsnop, D. R., and Jimenez, J. L.: Field-deployable, high-resolution, time-of-flight aerosol mass spectrometer, Anal. Chem., 78, 8281-8289, doi:10.1021/ac061249n, 2006.

Drewnick, F., Hings, S. S., DeCarlo, P., Jayne, J. T., Gonin, M., Fuhrer, K., Weimer, S., Jimenez, J. L., Demerjian, K. L., Borrmann, S. and Worsnop, D. R.: A New Time-of-Flight Aerosol Mass Spectrometer (TOF-AMS) - Instrument Description and First Field Deployment, Aerosol Sci. Tech., 39, 637658, doi:10.1080/02786820500182040, 2005.

Ehn, M., Junninen, H., Petäjä, T., Kurtén, T., Kerminen, V.-M., Schobesberger, S., Manninen, H. E., Ortega, I. K., Vehkamäki, H., Kulmala, M., and Worsnop, D. R.: Composition and temporal behavior of ambient ions in the boreal forest, Atmos. Chem. Phys., 10, 8513-8530, doi:10.5194/acp-10-8513-2010, 2010.

Ehn, M., Junninen, H., Schobesberger, S., Manninen, H. E., Franchin, A., Sipilä, M., Petäjä, T., Kerminen, V.-M., Tammet, H., Mirme, A., Mirme, S., Hõrrak, U., Kulmala, M., and Worsnop, D. R.: An Instrumental Comparison of Mobility and Mass Measurements of Atmospheric Small Ions, Aerosol Sci. Tech., 45, 522-532, doi:10.1080/02786826.2010.547890, 2011.

Ehn, M., Thornton, J. A., Kleist, E., Sipilä, M., Junninen, H., Pullinen, I., Springer, M., Rubach, F., Tillmann, R., Lee, B., LopezHilfiker, F., Andres, S., Acir, I.-H., Rissanen, M., Jokinen, T., Schobesberger, S., Kangasluoma, J., Kontkanen, J., Nieminen, T., Kurtén, T., Nielsen, L. B., Jørgensen, S., Kjaergaard, H. G., Canagaratna, M., Maso, M. D., Berndt, T., Petäjä, T., Wahner, A., Kerminen, V.-M., Kulmala, M., Worsnop, D. R., Wildt, J., and Mentel, T. F.: A large source of low-volatility secondary organic aerosol, Nature, 506, 476-479, doi:10.1038/nature13032, 2014.

Eisele, F. L.: Identification of tropospheric ions, J. Geophys. Res.Atmos., 91, 7897-7906, doi:10.1029/jd091id07p07897, 1986.

Faust, J. A., Junninen, H., Ehn, M., Chen, X., Ruusuvuori, K., Kieloaho, A.-J., Bäck, J., Ojala, A., Jokinen, T., Worsnop, D. R., Kulmala, M. and Petäjä, T.: Real-Time Detection of Arsenic Cations from Ambient Air in Boreal Forest and Lake Environments, Environ. Sci. Technol. Lett., 3, 42-46, doi:10.1021/acs.estlett.5b00308, 2016.

Friedman, B., Brophy, P., Brune, W. H., and Farmer, D. K.: Anthropogenic Sulfur Perturbations on Biogenic Oxidation: $\mathrm{SO}_{2}$ Additions Impact Gas-Phase $\mathrm{OH}$ Oxidation Products of $\alpha$ - and $\beta$-Pinene, Environ. Sci. Tech., 50, 1269-1279, doi:10.1021/acs.est.5b05010, 2016.

Heinritzi, M., Simon, M., Steiner, G., Wagner, A. C., Kürten, A., Hansel, A., and Curtius, J.: Characterization of the massdependent transmission efficiency of a CIMS, Atmos. Meas. Tech., 9, 1449-1460, doi:10.5194/amt-9-1449-2016, 2016.

Huey, L. G.: Measurement of trace atmospheric species by chemical ionization mass spectrometry: speciation of reactive nitrogen and future directions, Mass Spectrom. Rev., 26, 166-184, doi:10.1002/mas.20118, 2007.

Iyer, S., Lopez-Hilfiker, F., Lee, B. H., Thornton, J. A., and Kurtén, T.: Modeling the Detection of Organic and Inorganic Compounds Using Iodide-Based Chemical Ionization, J. Phys. Chem. A, 120, 576-587, doi:10.1021/acs.jpca.5b09837, 2016.

Jokinen, T., Sipilä, M., Junninen, H., Ehn, M., Lönn, G., Hakala, J., Petäjä, T., Mauldin III, R. L., Kulmala, M., and Worsnop,
D. R.: Atmospheric sulphuric acid and neutral cluster measurements using CI-APi-TOF, Atmos. Chem. Phys., 12, 4117-4125, doi:10.5194/acp-12-4117-2012, 2012.

Junninen, H., Ehn, M., Petäjä, T., Luosujärvi, L., Kotiaho, T., Kostiainen, R., Rohner, U., Gonin, M., Fuhrer, K., Kulmala, M., and Worsnop, D. R.: A high-resolution mass spectrometer to measure atmospheric ion composition, Atmos. Meas. Tech., 3, 10391053, doi:10.5194/amt-3-1039-2010, 2010.

Kim, S., Karl, T., Helmig, D., Daly, R., Rasmussen, R., and Guenther, A.: Measurement of atmospheric sesquiterpenes by proton transfer reaction-mass spectrometry (PTR-MS), Atmos. Meas. Tech., 2, 99-112, doi:10.5194/amt-2-99-2009, 2009.

Krechmer, J. E., Coggon, M. M., Massoli, P., Nguyen, T. B., Crounse, J. D., Hu, W., Day, D. A., Tyndall, G. S., Henze, D. K., Rivera-Rios, J. C., Nowak, J. B., Kimmel, J. R., Mauldin, R. L., Stark, H., Jayne, J. T., Sipilä, M., Junninen, H., Clair, J. M. S., Zhang, X., Feiner, P. A., Zhang, L., Miller, D. O., Brune, W. H., Keutsch, F. N., Wennberg, P. O., Seinfeld, J. H., Worsnop, D. R., Jimenez, J. L., and Canagaratna, M. R.: Formation of Low Volatility Organic Compounds and Secondary Organic Aerosol from Isoprene Hydroxyhydroperoxide Low-NO Oxidation, Environ. Sci. Tech., 49, 10330-10339, doi:10.1021/acs.est.5b02031, 2015.

Lee, B. H., Lopez-Hilfiker, F. D., Mohr, C., Kurtén, T., Worsnop, D. R., and Thornton, J. A.: An iodide-adduct high-resolution time-of-flight chemical-ionization mass spectrometer: application to atmospheric inorganic and organic compounds, Environ. Sci. Tech., 48, 6309-6317, doi:10.1021/es500362a, 2014.

Lopez-Hilfiker, F. D., Mohr, C., Ehn, M., Rubach, F., Kleist, E., Wildt, J., Mentel, Th. F., Lutz, A., Hallquist, M., Worsnop, D., and Thornton, J. A.: A novel method for online analysis of gas and particle composition: description and evaluation of a Filter Inlet for Gases and AEROsols (FIGAERO), Atmos. Meas. Tech., 7, 983-1001, doi:10.5194/amt-7-983-2014, 2014.

Lopez-Hilfiker, F. D., Mohr, C., Ehn, M., Rubach, F., Kleist, E., Wildt, J., Mentel, Th. F., Carrasquillo, A. J., Daumit, K. E., Hunter, J. F., Kroll, J. H., Worsnop, D. R., and Thornton, J. A.: Phase partitioning and volatility of secondary organic aerosol components formed from a-pinene ozonolysis and $\mathrm{OH}$ oxidation: the importance of accretion products and other low volatility compounds, Atmos. Chem. Phys., 15, 7765-7776, doi:10.5194/acp-15-7765-2015, 2015.

Lopez-Hilfiker, F. D., Iyer, S., Mohr, C., Lee, B. H., D’Ambro, E. L., Kurtén, T., and Thornton, J. A.: Constraining the sensitivity of iodide adduct chemical ionization mass spectrometry to multifunctional organic molecules using the collision limit and thermodynamic stability of iodide ion adducts, Atmos. Meas. Tech., 9, 1505-1512, doi:10.5194/amt-9-1505-2016, 2016.

Mohr, C., Lopez-Hilfiker, F. D., Zotter, P., Prévôt, A. S. H., Xu, L., Ng, N. L., Herndon, S. C., Williams, L. R., Franklin, J. P., Zahniser, M. S., Worsnop, D. R., Knighton, W. B., Aiken, A. C., Gorkowski, K. J., Dubey, M. K., Allan, J. D. and Thornton, J. A.: Contribution of nitrated phenols to wood burning brown carbon light absorption in Detling, United Kingdom during winter time, Environ. Sci. Tech., 47, 6316-6324, doi:10.1021/es400683v, 2013.

Olenius, T., Schobesberger, S., Kupiainen-Määttä, O., Franchin, A., Junninen, H., Ortega, I. K., Kurtén, T., Loukonen, V., Worsnop, D. R., Kulmala, M., and Vehkamäki, H.: Comparing simulated 
and experimental molecular cluster distributions, Faraday Discuss., 165, 75-89, doi:10.1039/c3fd00031a, 2013.

Rivera-Rios, J. C., Nguyen, T. B., Crounse, J. D., Jud, W., Saint Clair, J. M., Mikoviny, T., Gilman, J. B., Lerner, B. M., Kaiser, J. B., de Gouw, J., Wisthaler, A., Hansel, A., Wennberg, P. O., Seinfeld, J. H., and Keutsch, F. N.: Conversion of hydroperoxides to carbonyls in field and laboratory instrumentation: Observational bias in diagnosing pristine versus anthropogenically controlled atmospheric chemistry, Geophys. Res. Lett., 41, 86458651, doi:10.1002/2014g1061919, 2014.

Schobesberger, S., Franchin, A., Bianchi, F., Rondo, L., Duplissy, J., Kürten, A., Ortega, I. K., Metzger, A., Schnitzhofer, R., Almeida, J., Amorim, A., Dommen, J., Dunne, E. M., Ehn, M., Gagné, S., Ickes, L., Junninen, H., Hansel, A., Kerminen, V.-M., Kirkby, J., Kupc, A., Laaksonen, A., Lehtipalo, K., Mathot, S., Onnela, A., Petäjä, T., Riccobono, F., Santos, F. D., Sipilä, M., Tomé, A., Tsagkogeorgas, G., Viisanen, Y., Wagner, P. E., Wimmer, D., Curtius, J., Donahue, N. M., Baltensperger, U., Kulmala, M., and Worsnop, D. R.: On the composition of ammonia-sulfuric-acid ion clusters during aerosol particle formation, Atmos. Chem. Phys., 15, 55-78, doi:10.5194/acp-15-55-2015, 2015.

Sipilä, M., Sarnela, N., Jokinen, T., Junninen, H., Hakala, J., Rissanen, M. P., Praplan, A., Simon, M., Kürten, A., Bianchi, F., Dommen, J., Curtius, J., Petäjä, T., and Worsnop, D. R.: Bisulfate - cluster based atmospheric pressure chemical ionization mass spectrometer for high-sensitivity $(<100 \mathrm{ppqV})$ detection of atmospheric dimethyl amine: proof-of-concept and first ambient data from boreal forest, Atmos. Meas. Tech., 8, 4001-4011, doi:10.5194/amt-8-4001-2015, 2015.

Stark, H., Yatavelli, R. L. N., Thompson, S. L., Kimmel, J. R., Cubison, M. J., Chhabra, P. S., Canagaratna, M. R., Jayne, J. T., Worsnop, D. R., and Jimenez, J. L.: Methods to extract molecular and bulk chemical information from series of complex mass spectra with limited mass resolution, Int. J. Mass Spectrom., 389, 26-38, doi:10.1016/j.ijms.2015.08.011, 2015.

Veres, P., Roberts, J. M., Warneke, C., Welsh-Bon, D., Zahniser, M., Herndon, S., Fall, R., and de Gouw, J.: Development of negative-ion proton-transfer chemical-ionization mass spectrometry (NI-PT-CIMS) for the measurement of gas-phase organic acids in the atmosphere, Int. J. Mass Spectrom., 274, 48-55, doi:10.1016/j.ijms.2008.04.032, 2008.
Viidanoja, J., Reiner, T., and Arnold, F.: Laboratory investigations of negative ion molecule reactions of formic and acetic acids: implications for atmospheric measurements by ion-molecule reaction mass spectrometry, Int. J. Mass Spectrom., 181, 31-41, 1998.

Warneke, C., Veres, P., Murphy, S. M., Soltis, J., Field, R. A., Graus, M. G., Koss, A., Li, S.-M., Li, R., Yuan, B., Roberts, J. M., and de Gouw, J. A.: PTR-QMS versus PTR-TOF comparison in a region with oil and natural gas extraction industry in the Uintah Basin in 2013, Atmos. Meas. Tech., 8, 411-420, doi:10.5194/amt-8-4112015, 2015.

Wentzell, J. J. B., Liggio, J., Li, S.-M., Vlasenko, A., Staebler, R., Lu, G., Poitras, M.-J., Chan, T., and Brook, J. R.: Measurements of gas phase acids in diesel exhaust: a relevant source of HNCO?, Environ. Sci. Tech., 47, 7663-7671, doi:10.1021/es401127j, 2013.

Yatavelli, R. L. N., Lopez-Hilfiker, F., Wargo, J. D., Kimmel, J. R., Cubison, M. J., Bertram, T. H., Jimenez, J. L., Gonin, M., Worsnop, D. R., and Thornton, J. A.: A Chemical Ionization High-Resolution Time-of-Flight Mass Spectrometer Coupled to a Micro Orifice Volatilization Impactor (MOVI-HRToF-CIMS) for Analysis of Gas and Particle-Phase Organic Species, Aerosol Sci. Tech., 46, 1313-1327, doi:10.1080/02786826.2012.712236, 2012.

Yatavelli, R. L. N., Stark, H., Thompson, S. L., Kimmel, J. R., Cubison, M. J., Day, D. A., Campuzano-Jost, P., Palm, B. B., Hodzic, A., Thornton, J. A., Jayne, J. T., Worsnop, D. R., and Jimenez, J. L.: Semicontinuous measurements of gas-particle partitioning of organic acids in a ponderosa pine forest using a MOVI-HRToFCIMS, Atmos. Chem. Phys., 14, 1527-1546, doi:10.5194/acp14-1527-2014, 2014.

Zhao, R., Mungall, E. L., Lee, A. K. Y., Aljawhary, D., and Abbatt, J. P. D.: Aqueous-phase photooxidation of levoglucosan a mechanistic study using aerosol time-of-flight chemical ionization mass spectrometry (Aerosol ToF-CIMS), Atmos. Chem. Phys., 14, 9695-9706, doi:10.5194/acp-14-9695-2014, 2014. 